\title{
Human duct cells contribute to $\beta$ cell compensation in insulin resistance
}

\author{
Ercument Dirice, ${ }^{1,2}$ Dario F. De Jesus, ${ }^{1,2,3}$ Sevim Kahraman, ${ }^{1,2}$ Giorgio Basile, ${ }^{1,2}$ Raymond W.S. Ng, \\ Abdelfattah El Ouaamari, ${ }^{1,2}$ Adrian Kee Keong Teo, ${ }^{1,2}$ Shweta Bhatt, ${ }^{1,2}$ Jiang Hu, ${ }^{1,2}$ \\ and Rohit N. Kulkarni $\mathbf{i}^{1,2,4}$ \\ 'Section of Islet Cell and Regenerative Biology, Joslin Diabetes Center, Boston, Massachusetts, USA. ${ }^{2}$ Department of \\ Medicine, Brigham and Women's Hospital, Harvard Medical School, Boston, Massachusetts. USA. ${ }^{3}$ Graduate Program \\ in Areas of Basic and Applied Biology, Abel Salazar Biomedical Sciences Institute, University of Porto, Porto, Portugal. \\ ${ }^{4}$ Harvard Stem Cell Institute, Boston, Massachusetts, USA.
}

\begin{abstract}
The identification of new sources of $\beta$ cells is an important endeavor with therapeutic implications for diabetes. Insulin resistance, in physiological states such as pregnancy or in pathological states such as type 2 diabetes (T2D), is characterized by a compensatory increase in $\beta$ cell mass. To explore the existence of a dynamic $\beta$ cell reserve, we superimposed pregnancy on the liver-specific insulin receptor-KO (LIRKO) model of insulin resistance that already exhibits $\beta$ cell hyperplasia and used lineage tracing to track the source of new $\beta$ cells. Although both control and LIRKO mice displayed increased $\beta$ cell mass in response to the relative insulin resistance of pregnancy, the further increase in mass in the latter supported a dynamic source that could be traced to pancreatic ducts. Two observations support the translational significance of these findings. First, NOD/SCID- $\gamma$ LIRKO mice that became pregnant following cotransplantation of human islets and human ducts under the kidney capsule showed enhanced $\beta$ cell proliferation and an increase in ductal cells positive for transcription factors expressed during $\beta$ cell development. Second, we identified duct cells positive for immature $\beta$ cell markers in pancreas sections from pregnant humans and in individuals with T2D. Taken together, during increased insulin demand, ductal cells contribute to the compensatory $\beta$ cell pool by differentiation/neogenesis.
\end{abstract}

Authorship note: DFDJ and SK contributed equally to this work.

Conflict of interest: The authors have declared that no conflict of interest exists.

Copyright: (c) 2019 American Society for Clinical Investigation

Submitted: January 2, 2018

Accepted: March 5, 2019

Published: April 18, 2019

Reference information: /CI Insight. 2019;4(8):e99576. https://doi. org/10.1172/jici.insight.99576.

\section{Introduction}

A fine balance between cell growth (replication, hypertrophy, neogenesis) and cell loss (apoptosis, atrophy, autophagy) maintains an optimal $\beta$ cell mass to appropriately regulate glycemia in adult mammals. Compensatory changes in 1 or more of these processes underlie the ability of $\beta$ cells to dynamically adapt to physiological or pathophysiological states or to injury (1-8). Among these, pregnancy, characterized by a temporary increase in insulin demand, is a physiological example of dynamic $\beta$ cell mass expansion in both rodents and humans (1-3). Indeed rodents exhibit up to a 4-fold increase in $\beta$ cell mass during pregnancy that remarkably reverts to normal in the postpartum period $(4,5)$.

The ability of $\beta$ cell mass to dynamically adapt during pregnancy has prompted investigators to investigate the mechanism(s) underlying this process and identify the signals that contribute to increasing insulin-secreting cells, with the long-term goal of developing approaches to treat both type 1 and type 2 diabetes (T2D). Several processes, including hypertrophy (5), increase in proliferation during pregnancy $(5,9,10)$, followed by a decrease in $\beta$ cell proliferation and size, and increased apoptosis in the postpartum period (4), which have been implicated to underlie the dynamic changes in $\beta$ cell mass during pregnancy. The hormones contributing to this increased $\beta$ cell mass include prolactin and placental lactogen $(11,12)$, hepatocyte nuclear factor 4 (13), and HGF (14). These hormones are mediated by downstream signals, such as STAT5 $(15,16)$ or serotonin $(17)$, and proteins in the insulin/IGF-1 signaling pathway, such as MAPK (18), PI3K, and insulin receptor substrates 1/2 (11).

The source of new cells that contribute to the expansion of adult $\beta$ cell mass during adaptive processes has been debated for several years. Although in vitro and in vivo experiments suggest acinar cells can be converted into $\beta$-like cells (19-21), lineage trace experiments did not support this hypothesis in vivo (22). Adult ductal cells have been suggested to act as precursors of $\beta$ cells because embryonic ducts are direct precursors of neurogenin $3^{+}\left(\mathrm{NGN3}^{+}\right)$cells, which can give rise to all pancreatic endocrine cells (23). How- 
ever, Dor et al. reported that $\beta$ cell mass in adult mice is derived mostly from replication (24), a conclusion that was further supported by a sequential, thymidine analog-based lineage-tracing approach that indicated regeneration of adult $\beta$ cells does not involve specialized progenitors (25). Moreover, Xiao X et al. reported that $\mathrm{NGN3}^{+}$cells within the ducts do not differentiate to $\beta$ cells (26), which is supported by a previous report (27). On the other hand, an independent lineage-tracing study showed that ductal cells, marked using a transgene driven by the carbonic anhydrase II promoter, gave rise to $\beta$ cells after birth and following ductal ligation in older mice (28). Thus, the contribution of non- $\beta$ cell sources to $\beta$ cell mass in adult organisms, especially in humans, continues to be an active area of research $(3,23,29-34)$.

To directly address this issue, we took advantage of a unique model, the liver-specific insulin receptor$\mathrm{KO}$ (LIRKO) mouse, which adapts to insulin resistance by forming hyperplastic functional islets, and asked whether these mice are able to further increase the number of $\beta$ cells upon superimposition of pregnancy. Interestingly, the LIRKO animals continued to expand their $\beta$ cell mass in the endogenous pancreas even during pregnancy beyond that observed in the nonpregnant state, and lineage tracing pointed to the duct as a source of $\beta$ cells. Pregnant LIRKO mice cotransplanted with human ducts and human islets showed an increase in insulin ${ }^{+}$ductal cells, providing translational significance. Finally, analysis of pancreas sections from humans during different trimesters of pregnancy and from patients with T2D revealed significantly greater insulin ${ }^{+}$ductal cells and smaller islet clusters compared with controls. Together, these data provide genetic and translational evidence for neogenesis as a source of $\beta$ cell compensation in the adaptation to physiological or pathophysiological states in humans.

\section{Results}

Glucose homeostasis and metabolic parameters in pregnant LIRKO mice. During late pregnancy, mothers develop relative insulin resistance to accommodate an appropriate supply of nutrients to the fetus (12). To assess whether superimposing pregnancy in insulin-resistant LIRKO (35) mice is able to activate generation of new $\beta$ cells from additional reserves, we phenotyped the animals before, during, and after pregnancy. Although both control and LIRKO mice gained significant weight during the progression of pregnancy/gestation $(G)$, no significant differences were observed between groups before or during pregnancy or in the postpartum (P) periods (Supplemental Figure 1A; supplemental material available online with this article; https://doi. org/10.1172/jci.insight.99576DS1). As expected, serum insulin levels in control mice increased during pregnancy, resulting in reduced blood glucose levels (Figure 1, A and B). The higher circulating insulin levels in nonpregnant (G0) LIRKO mice further increased and remained consistently higher during pregnancy, with elevated blood glucose levels peaking on day G15.5 and returning to normal levels on P4 (Figure 1, A and B).

Next, examination of acute-phase insulin release in response to oral glucose showed a relatively higher insulin secretion in pregnant LIRKO mice on G15.5 (Supplemental Figure 1B) that was consistent with their increased $\beta$ cell mass (35). In addition, the impaired glucose tolerance in nonpregnant LIRKO mice worsened around midpregnancy (G15.5) (Figure 1C and Supplemental Figure 1C). The LIRKO mice also exhibited a relatively severer insulin resistance compared with controls in both nonpregnant and pregnant states (Figure 1D and Supplemental Figure 1D), consistent with our previous report (36), supporting the notion that the pregnant LIRKO mouse is a suitable model to investigate pathways that contribute to expanding the $\beta$ cell pool during extreme demands.

LIRKO mice exhibit ability to further enhance proliferation of $\beta$ cells during pregnancy. To evaluate the effects of superimposing pregnancy in a genetic model that already exhibits compensatory islet hyperplasia, we systematically examined islet architecture, islet cell composition, and $\beta$ cell proliferation, size, mass, and apoptosis. Analysis of islet morphology in the pancreas sections of control pregnant or nonpregnant mice showed a relatively normal islet cell distribution pattern with a core of $\beta$ cells and non- $\beta$ cells forming the mantle (Figure 1E). In contrast, and especially in pregnant LIRKO mice, the core and majority (90\%-95\%) of the periphery of the hyperplastic islets were almost exclusively composed of $\beta$ cells, with a lower percentage of $\alpha$ and $\delta$ cells accumulated in one-half of the islet (Figure 1E).

Analyses of cells within each islet showed a dramatically increased $\beta$ cell number in both nonpregnant (G0) and pregnant LIRKO mice (Figure 1F). Furthermore, the islet endocrine cell composition revealed that, although the $\beta$ cell population was further increased in pregnant mice in both groups, the proportions of glucagon- and somatostatin-producing cells were significantly lower during and following pregnancy only in the LIRKO mice (Figure 1G). The increase in $\beta$ cells from G0 to G15.5 in control mice was secondary to enhanced proliferation as shown by an increase in $\mathrm{Ki}^{+} 7^{+} \beta$ cells followed by a decrease to nonpregnant levels 
after birth (Figure 1, H and I, and Supplemental Table 1). In contrast, the approximately 2-fold increase in $\beta$ cell proliferation in LIRKO mice, compared with control animals at G0, went up further, approximately 4-fold, by G15.5 and decreased to control levels postpartum (P0 and P4) (Figure 1, H and I, and Supplemental Table 1). The $\beta$ cell proliferation was confirmed by immunostaining for the $\mathrm{M}$ phase proliferation marker, phosphohistone H3 (pHH3) (Supplemental Figure 2, A and B, and Supplemental Table 1), and was consistent with the total $\beta$ cells per $\mathrm{mm}^{2}$ of pancreas area (Supplemental Figure 2C). Although $\alpha$ cell proliferation showed a decrease in pregnant control mice, the LIRKO mice exhibited significantly lower $\alpha$ cell proliferation before, during, and after pregnancy (Supplemental Figure 2, D and E) consistent with the islet cell composition in LIRKO mice shown in Figure 1G. Whether this decrease in $\alpha$ cells is because some of them are contributing to the increased $\beta$ cell pool during pregnancy requires further investigation.

Similar to the effects on proliferation, $\beta$ cell mass and area increased during pregnancy in both groups and decreased postpartum compared with nonpregnant states. In striking contrast, pregnant LIRKO mice showed an additional significant increase in $\beta$ cell mass and area after G15.5 that peaked at P0 compared with controls at the same time point (Figure 1, J and K, and Supplemental Figure 2F). Furthermore, cell size assessed by coimmunostaining for $\beta$-catenin and insulin did not show statistically significant differences between control and LIRKO mice before, during, or after pregnancy (Supplemental Figure 2, G and H). $\beta$ Cell apoptosis evaluated by TUNEL-labeling did not show differences between control and LIRKO mice at G15.5 (Supplemental Figure $2 \mathrm{I})$. Overall, these results suggest that in addition to replication, LIRKO mice mobilize non- $\beta$ cells to further enhance $\beta$ cell numbers during pregnancy, and this prompted us to speculate about other sources of $\beta$ cells.

Alterations in pregnancy hormones and serotonin in LIRKO mice. Pregnancy hormones have been a focus of study as important stimuli to drive $\beta$ cell proliferation during pregnancy (18). Surprisingly, prolactin and progesterone levels were significantly decreased in LIRKO mice during pregnancy while only estradiol levels were increased at G15.5 in LIRKO compared with control mice (Supplemental Figure 3, A-C). Despite the dramatic difference in pregnancy hormones, the duration of pregnancy and litter sizes were similar between groups (36). Furthermore, the levels of serotonin, a neurotransmitter that has been implicated in pregnancy hormone-induced increase in $\beta$ cell mass (17), was higher in the LIRKO mice compared with control mice (Supplemental Figure 4, A-G). Although we did not observe differences in expression of serotonin receptors between groups (data not shown), additional work is necessary to explore the role of serotonin, if any, in promoting an increase in $\beta$ cell mass in the LIRKO model.

Increased insulin ${ }^{+}$ductal cells and small islet clusters adherent to ducts in pregnant LIRKO mice. To investigate the direct role of ductal cells in the expansion of $\beta$ cell mass, we first examined the incidence of cells that were double positive for insulin and the duct marker, Dolichos biflorus agglutinin (DBA). Control mice showed an increase in insulin and DBA double-positive cells during pregnancy that reduced to nonpregnant levels in the postpartum period (Figure 2, A and B). Although LIRKO dams revealed a similar pattern, the number of insulin ${ }^{+}$cells in the duct epithelium was significantly higher during and after the first 4 days postpartum (Figure 2, A and B). Notably, glucagon ${ }^{+}$ductal cells were also significantly higher at G17.5 in pregnant LIRKO mice (Supplemental Figure 5A). It is possible that the increase in DBA/glucagon double-positive cells represents a compensation for reduced $\alpha$ cell content during late pregnancy (Figure 1G).

Neogenesis is characterized by an increase in the number of small islet clusters (37). Indeed, we also observed an increase in scattered small islet clusters before, during, and after pregnancy in LIRKO mothers that reached significance at G15.5 (control: 0.2 scattered islets $/ \mathrm{mm}^{2}$ vs. LIRKO: 0.6 scattered islets $/ \mathrm{mm}^{2}, P=0.008$ ) (Supplemental Figure $5 \mathrm{~B}$ ). We also observed islet clusters that appeared to be virtually adherent to ducts in the LIRKO mice, especially at G15.5 (G0: 10.1\% control vs. 21.2\% LIRKO, $P=0.06$; and G15.5: $10.5 \%$ control vs. $37.8 \%$ LIRKO, $P=0.002$ of total small islet clusters, respectively) (Figure 2, C and D). The percentage of small islet clusters that were located less than or equal to $5 \mu \mathrm{m}$ from ducts was higher in LIRKO mice during the transition from G17.5 to P4 (Figure 2D). These data suggest that neogenesis is a contributor to the compensatory $\beta$ cell pool.

The duct epithelium contributes to islet endocrine cell formation. The lack of a phenotype in animals with $\mathrm{KO}$ of insulin receptors in ducts (S. Bonner-Weir, Joslin Diabetes Center, personal communication) encouraged us to use duct-specific, carbonic anhydrase-driven Cre and floxed insulin receptor mice to develop an inducible lineage trace model to directly examine whether duct cells are a source of new $\beta$ cells. To this end we bred tamoxifen-inducible (TM-inducible) carbonic anhydrase II-CreER ${ }^{T M} R 26 R$-eYFP (reporter-enhanced yellow fluorescence protein, eYFP) (38) mice with LIRKO animals (Figure 3A). Offspring, carrying the carbonic anhydrase II-driven (CAII-driven) Cre transgene and homozygous for both eYFP and loxP-targeted insulin 

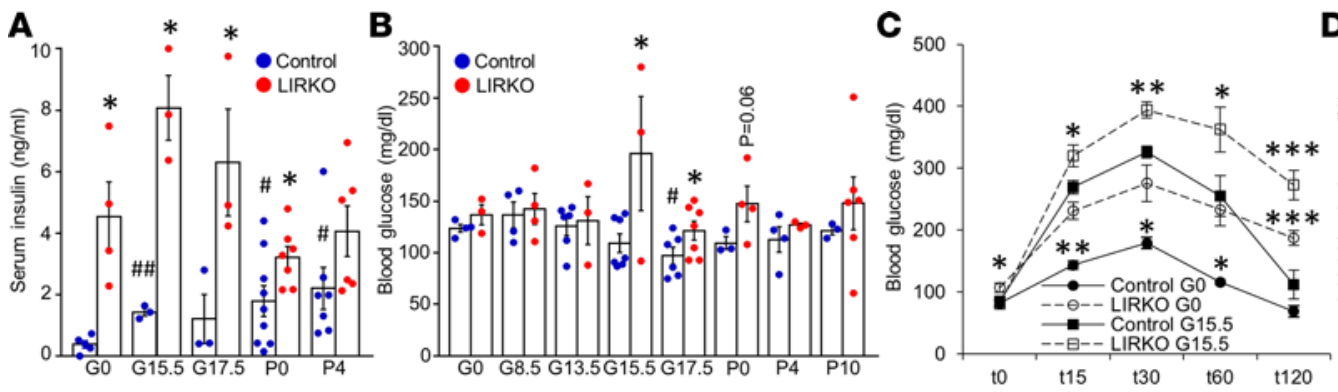

E
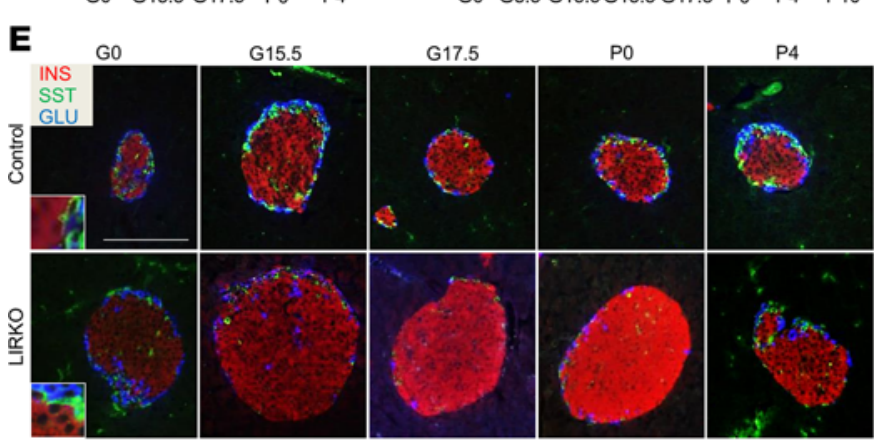

$\mathbf{F}$
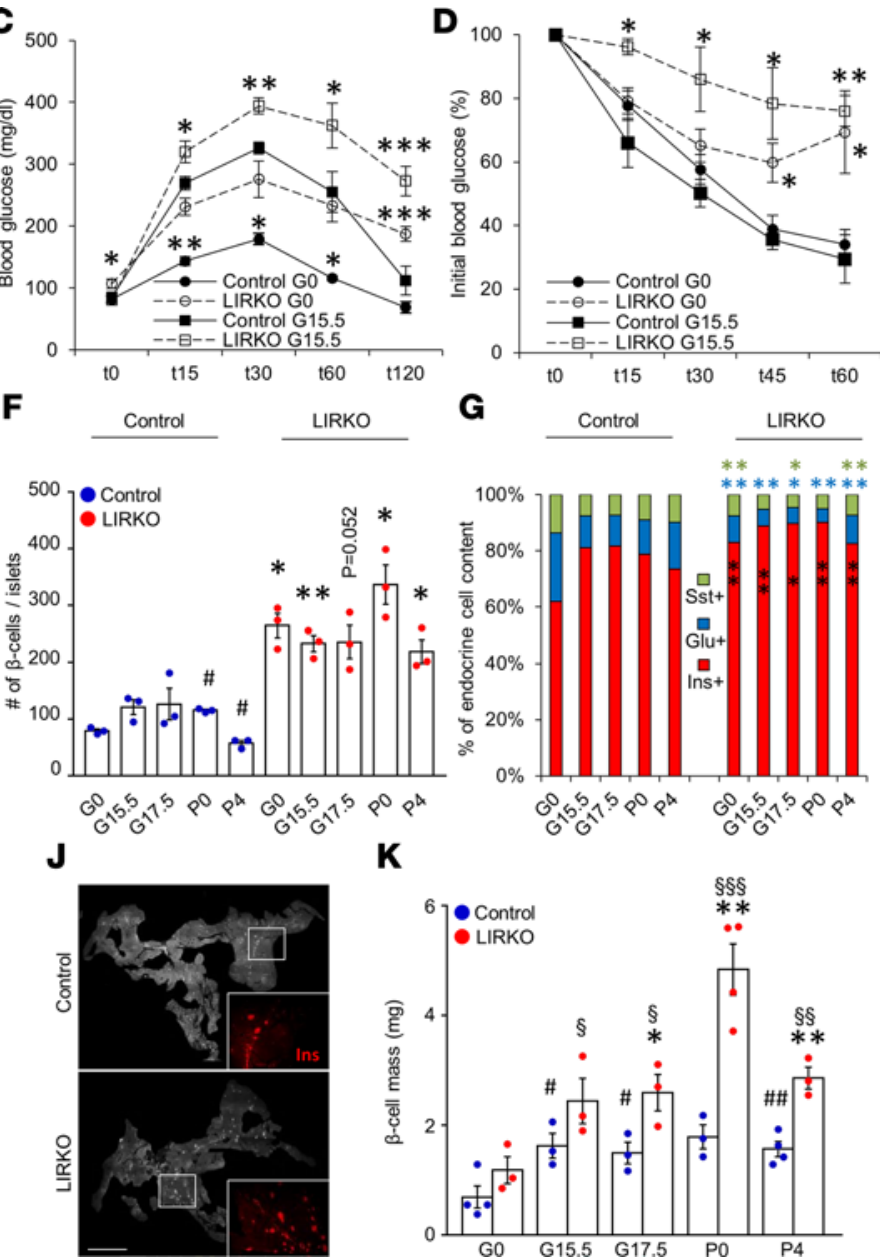

G

Control
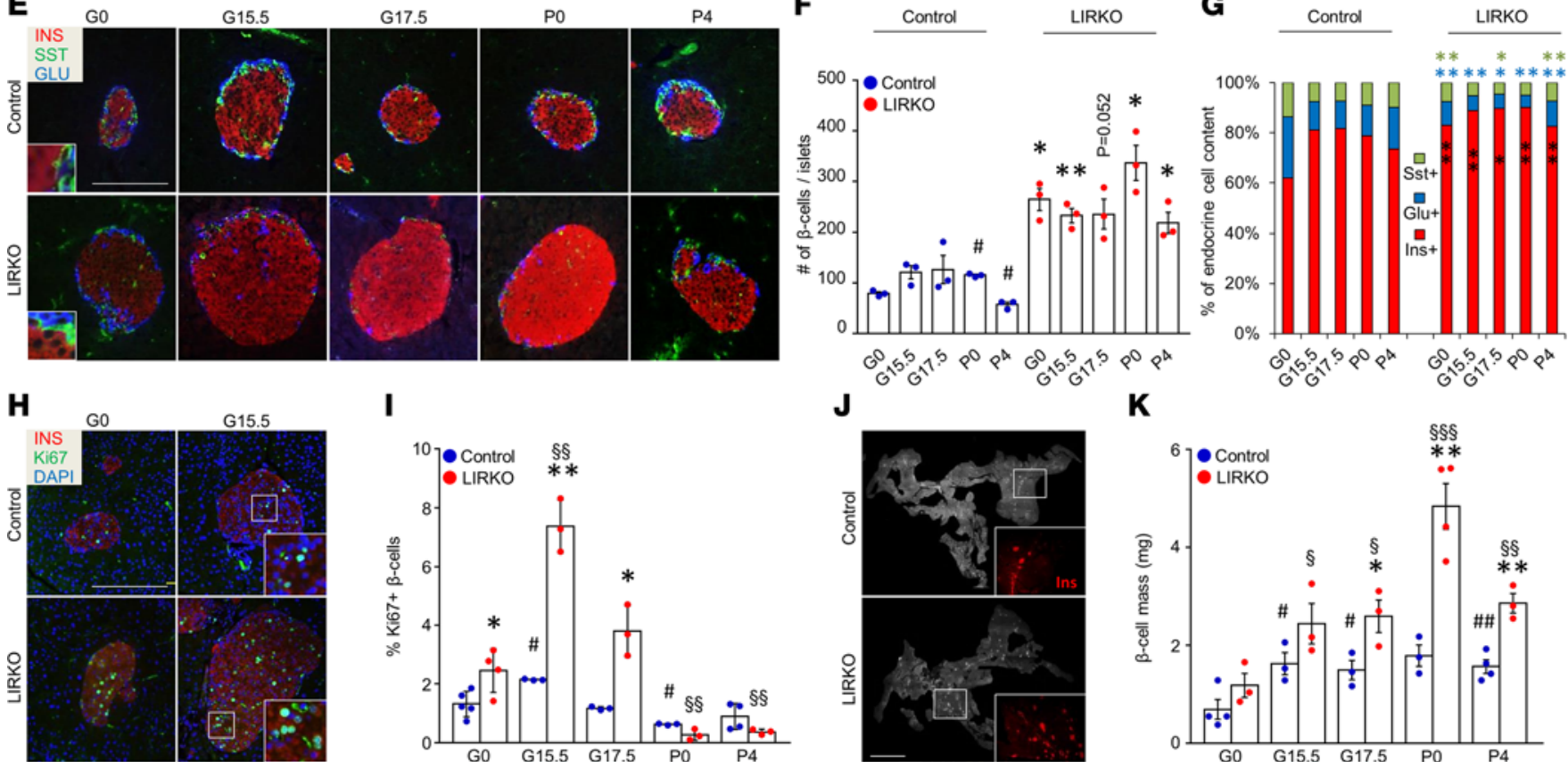

I

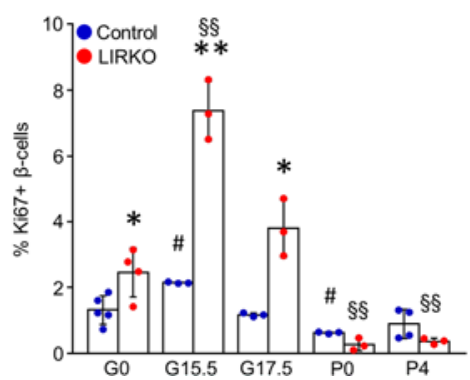

$K$
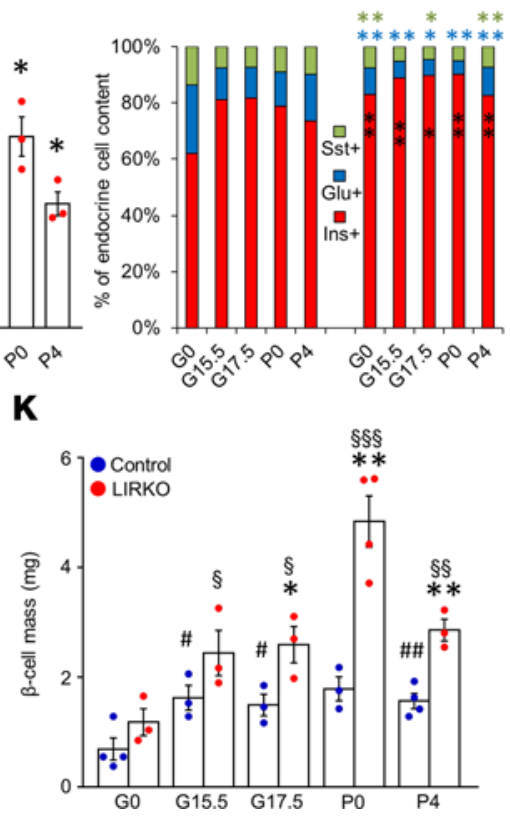

Figure 1. Compensatory islet hyperplasia in pregnant LIRKO mice. (A) Serum insulin ( $n=3-9$ mice per group, 2-tailed Student's $t$ test) and (B) blood glucose levels ( $n=3-7$ mice per group, 2-tailed Student's $t$ test) in female control and LIRKO mice measured before gestation (C0), during (C15.5, G17.5) pregnancy, and after (P4 and P10) pregnancy. (C) Blood glucose values following an oral glucose tolerance test (2.5 g/kg BW) ( $n=4-7$ mice per group, 2-tailed Student's $t$ test) and (D) glucose levels plotted as percentage of basal values, following i.p. injection of insulin ( $1 \mathrm{U} / \mathrm{kg} \mathrm{BW)} \mathrm{(} n=3-6 \mathrm{mice}$ per group, 2-tailed Student's $t$ test). Solid line indicates control, and dashed line indicates LIRKO mice. Nonpregnant mice are shown as circles and pregnant mice as squares. (E) Representative immunofluorescence images of pancreatic sections stained with a cocktail of antibodies against insulin (shown in red), glucagon (shown in blue), and somatostatin (shown in green) as described in Methods. Scale bar: $100 \mu \mathrm{m}$. Original magnification, $\times 20$. Insets show enlarged endocrine cells. (F) Average number of $\beta$ cells per islet. A total of 20 randomly selected islets were analyzed per group for all time points $(n=3$ mice per group, 2-tailed Student's $t$ test). (G) Quantification of the islet endocrine cell content. $\alpha, \beta$, and $\delta$ cell numbers were counted per islet, and 20 randomly selected islets were analyzed per mouse in each group for all time points and presented as the percentage of total islet endocrine cells ( $n=3$ mice per group, 2-tailed Student's $t$ test). (H) Representative images of pancreatic sections obtained from nonpregnant and pregnant (G15.5) control and LIRKO mice stained for insulin (red), proliferation marker Ki67 (green), and nuclear marker DAPI (blue). Insets point to Ki67 cells. Scale bar: $100 \mu \mathrm{m}$. (I) Quantification of Ki67 $\beta$ cells ( $n=3-5$ mice per group, 2-tailed Student's $t$ test) (for quantification, see Supplemental Table 1) (J) Representative pancreas sections with insets showing insulin ${ }^{+}(\mathrm{red})$ islets. Scale bar: $4 \mathrm{~mm}$. (K) Morphometric analysis of $\beta$ cell mass as described in Methods ( $n=3-4$ mice per group, 2-tailed Student's $t$ test). Scale bars: $100 \mu \mathrm{m}$ (A and B), $4 \mathrm{~mm}$ (J). ${ }^{*}$ Control versus control, ${ }^{*}$ control versus LIRKO, and ${ }^{~}$ LIRKO versus LIRKO. Data are expressed as mean \pm SEM. ${ }^{\#} P, \$ P$, and ${ }^{*} P<0.05 ;{ }^{\#} P, \$ \S P$, and ${ }^{* *} P<0.01$; and $\$ \$ \$ P$ and ${ }^{* *} P<0.001$.

receptor, were grouped based on the absence (Lox-YFP) or presence (LIRKO-YFP) of the albumin promoter-driven Cre transgene, respectively. Because animals receiving either continuous or 5-day injections of TM during pregnancy had spontaneous abortions, we limited the injections to 3 days $(100 \mathrm{mg} / \mathrm{kg})$ starting at G10.5 followed by harvesting of pancreases on day G15.5. Untreated groups were included as controls, and no $\mathrm{YFP}^{+}$staining was detectable. As expected, the labeling efficiency was relatively low in the TM-treated group; nevertheless, confocal microscopy of samples from TM-treated pregnant and nonpregnant Lox-YFP and LIRKO-YFP mice demonstrated YFP-marked, insulin ${ }^{+}$cells in pancreatic islets that were localized close to ductal epithelium (Figure 3B). Control pregnant mice (G15.5) exhibited increased YFP-marked, insulin ${ }^{+}$ cells compared with nonpregnant animals (Figure $3 \mathrm{C}$ ), and this number was further increased in pregnant LIRKO-YFP (by approximately 2.3-fold vs. pregnant Lox-YFP, $P=0.07$; and by approximately 5-fold vs. 
A
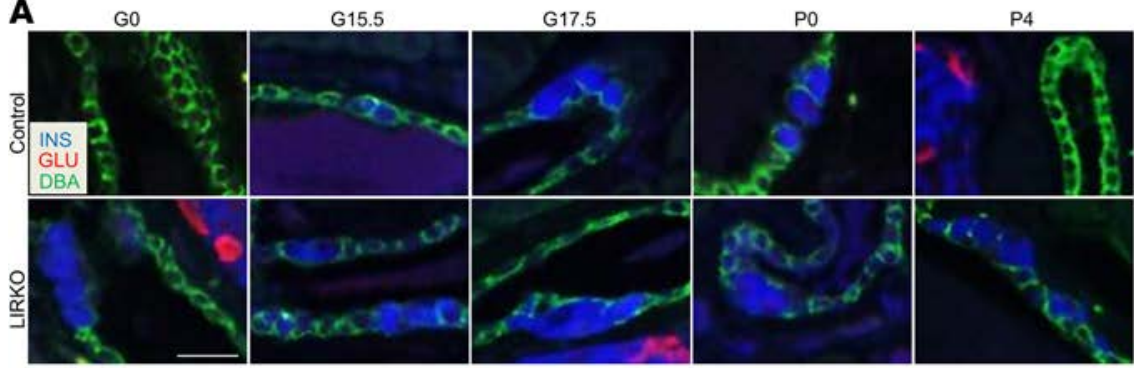

B

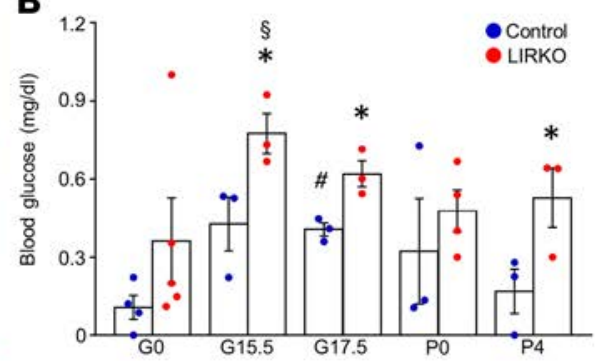

G15.5
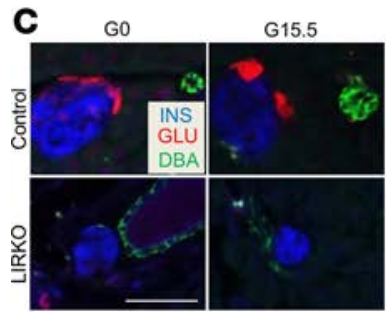

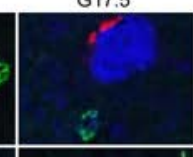

PO

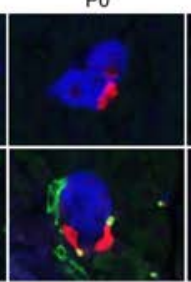

P4

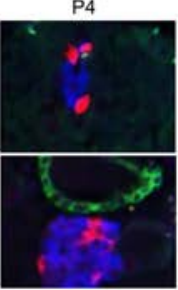

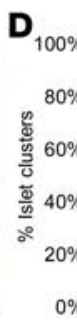

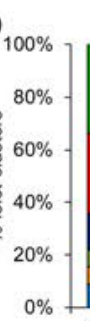

Control

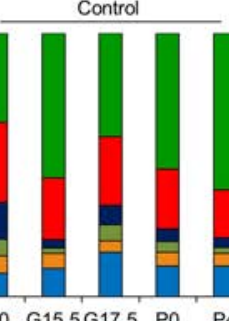

LIRKO

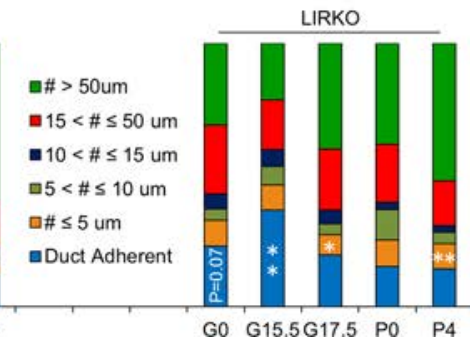

Figure 2. Insulin+ ductal cells and small islet clusters localized close to ducts are increased in LIRKO mice during pregnancy. (A) Representative immunofluorescence images obtained from control and LIRKO mice before, during, and after pregnancy and stained for insulin (shown in blue), glucagon (shown in red) and ductal marker DBA (shown in green). Insets point to DBA and insulin ${ }^{+}$cells. (B) Quantification of DBA and insulin double-positive cells in pregnant and nonpregnant control and LIRKO mice ( $n=3-5$ mice per group, 2-tailed Student's $t$ test). (C) Representative immunofluorescence images showing small islet clusters positive for insulin (blue) or glucagon (red) and DBA (green). Insets point to clusters localized to ductal structure. (D) Quantification of the distance of scattered islet clusters (fewer than 10 islet cells) from the ducts. Groups are shown as percentage of total scattered islets that were counted ( $n=3$ pancreas sections analyzed per group for each time point, 2-tailed Student's $t$ test). Scale bars: $10 \mu \mathrm{m}$ (A), $20 \mu \mathrm{m}$ (C). " Control versus control, ${ }^{*}$ control versus LIRKO, and ${ }^{\S}$ LIRKO versus LIRKO. Data are expressed as mean $\pm \mathrm{SEM}$. ${ }^{*} P$, ${ }^{\S} P$, and ${ }^{*} P<0.05$; ${ }^{* *} P<0.01$.

nonpregnant LIRKO-YFP mice, $P=0.03$ ), providing direct genetic evidence of ductal contribution to new $\beta$ cells. Although we did not find any insulin ${ }^{+}, \mathrm{YFP}^{+}$, or SOX9 ${ }^{+}$cells in G0 and G15.5 Lox-YFP mice, the data in the lineage-tracing studies were supported by the observation of insulin ${ }^{+}, \mathrm{YFP}^{+}$and $\mathrm{SOX}^{+}$ductal cells in pregnant LIRKO-YFP mice and insulin and SOX9 double-positive ductal cells in nonpregnant LIRKO-YFP animals (Supplemental Figure 6A). Moreover, YFP and pancreas/duodenum homeobox protein 1 (PDX1) immunostaining revealed double-positive islet $\beta$ cells only in LIRKO-YFP mice and were observed to be higher during pregnancy (G15.5) (Supplemental Figure 6B). The specificity to generate only $\beta$ cells by lineage tracing was confirmed by lack of coexpression of YFP and $\alpha$ cell markers (Supplemental Figure 6C).

Human $\beta$ cell proliferation is increased in response to pregnancy in NOD/SCID- $\gamma$ LIRKO mice transplanted with human islets. To examine the translational significance of the dramatic increase in $\beta$ cell mitosis in pregnant LIRKO mice (G15.5), we crossed LIRKO animals with immunodeficient NOD/SCID- $\gamma$ (NSG) mice (Figure 4A), to allow for human tissue engraftment (39). Offspring carrying albumin promoter-driven Cre transgene (NSG-LIRKO mice) continued to exhibit an insulin-resistant phenotype that was similar to the original LIRKO phenotype (Supplemental Figure 7, A-F, and ref. 40). Next, female NSG-Lox (control) and NSGLIRKO mice were rendered pregnant following transplantation of human islets (for donor information, see Supplemental Table 2) under the kidney capsule (Figure 4A and Supplemental Figure 8A), and the islet grafts bearing kidneys and endogenous pancreases were harvested at G15.5. The detection of human insulin and C-peptide in mouse serum confirmed graft survival and functioning human islets at tissue harvest. The average human insulin values (all approximate) were NSG-Lox: $3 \mathrm{mU} / \mathrm{L}$ (G0) and $8 \mathrm{mU} / \mathrm{L}$ (G15.5); NSGLIRKO: $39 \mathrm{mU} / \mathrm{L}$ (G0) and $116 \mathrm{mU} / \mathrm{L}$ (G15.5). The average human C-peptide values (all approximate) were NSG-Lox: $102 \mathrm{mU} / \mathrm{L}$ (G0) and $297 \mathrm{mU} / \mathrm{L}$ (G15.5); NSG-LIRKO: $863 \mathrm{mU} / \mathrm{L}$ (G0) and $497 \mathrm{mU} / \mathrm{L}$ (G15.5). Analysis of BrdU incorporation and Ki67 immunostaining revealed a significant increase in human $\beta$ cell proliferation in islet grafts obtained from pregnant NSG-Lox mice compared with nonpregnant controls (Figure 4, B-D; Supplemental Figure 8, A and B; and Supplemental Table 3). The level of human $\beta$ cell proliferation in nonpregnant NSG-LIRKO mice was comparable to pregnant NSG-Lox mice, whereas a significant $(P=$ 0.04 ) increase was evident in pregnant NSG-LIRKO mice compared with their corresponding controls (pregnant NSG-Lox) (Figure 4, B-D; Supplemental Figure 8, A and B; and Supplemental Table 3). Similar results 
A
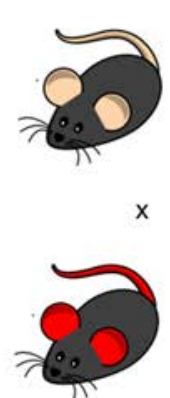

ARKO

Alb-CRE IR $R^{\text {loxlox }}$
G0

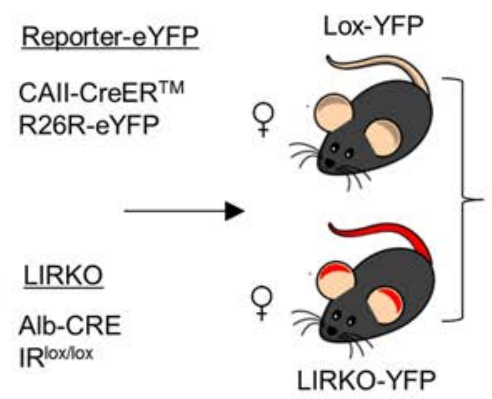

G0.5

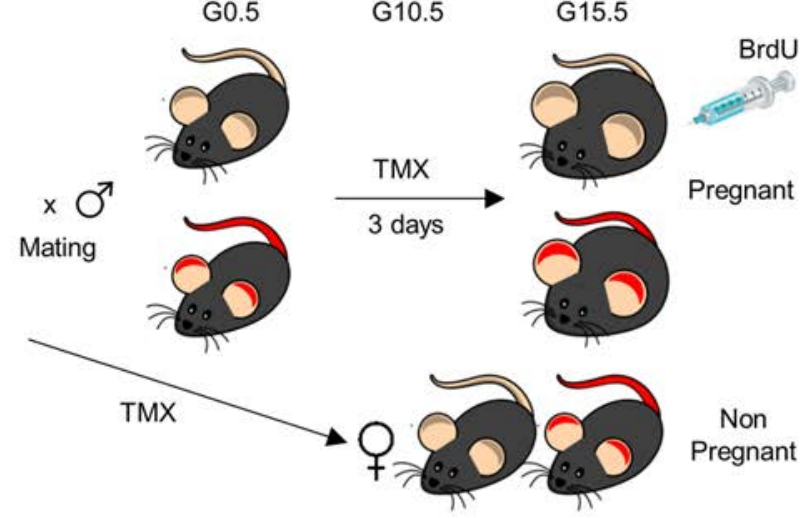

B
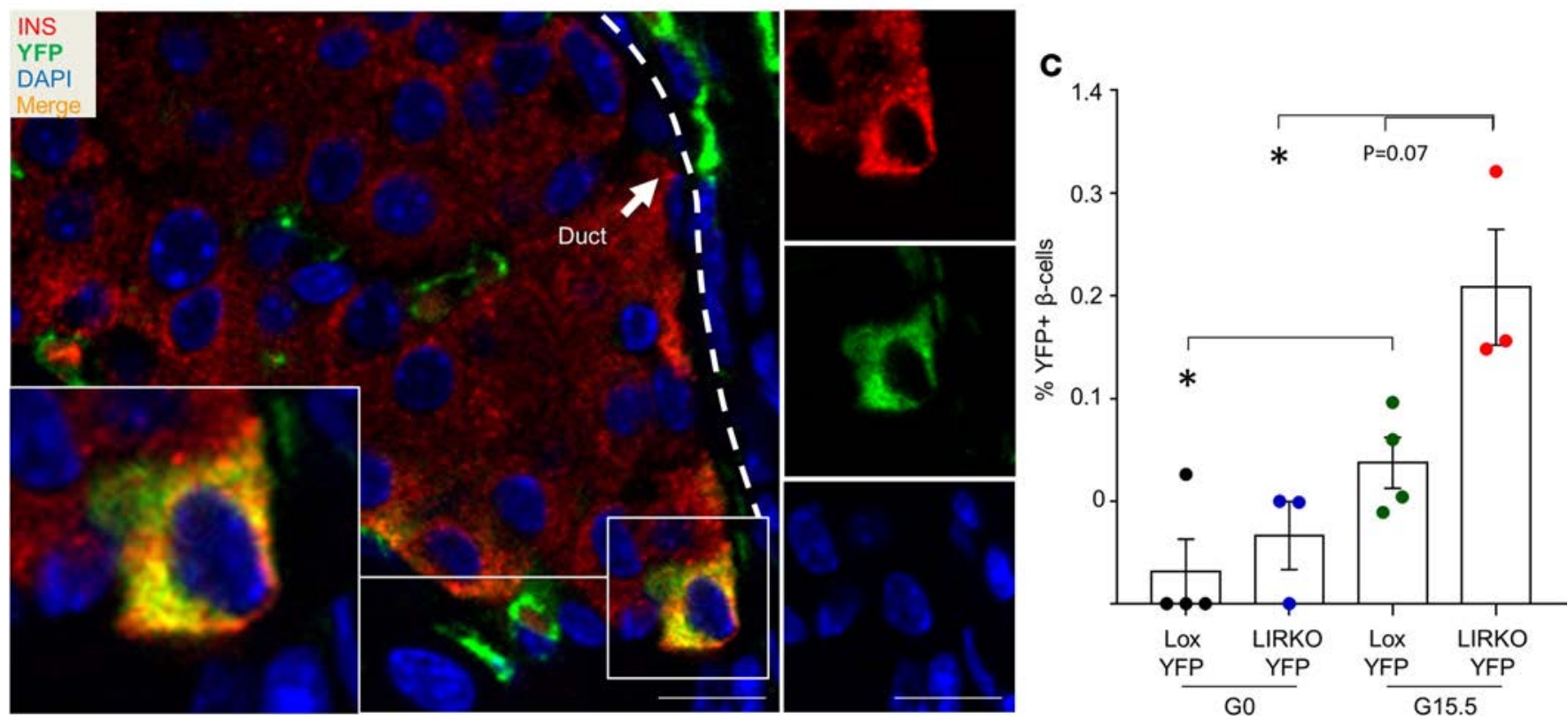

Figure 3. The duct epithelium contributes to islet endocrine cell formation. (A) Experimental strategy showing CAll-CreER ${ }^{\mathrm{TM}} R 26 R$-eYFP mice crossed to $A / b$-Cre InsR $R^{f / f l}$ animals to generate Lox-YFP and LIRKO-YFP mice and subsequently rendered pregnant. Pancreas was harvested at G15.5 after TM injection on G10.5 for 3 consecutive days. (B) Representative confocal image of pancreas section obtained from TM-treated pregnant LIRKOYFP mice stained for insulin (shown in red), YFP (shown in green), and DAPI (shown in blue). Individual images for 1 cell are shown in the upper, middle, and bottom right. Inset shows YFP-marked, insulin+ (coexpression, yellow) $\beta$ cell within the islet. White arrow pointing to the dashed white line indicates ductal structures close to the islet. Scale bar: $10 \mu \mathrm{m}$. (C) Quantification of YFP+ $\beta$ cells ( $n=3-4$ mice per group, 2-tailed Student's $t$ test). Data are expressed as mean \pm SEM. ${ }^{*} P<0.05$.

were observed with the proliferation marker, pHH3 (Supplemental Figure 8C and Supplemental Table 3). Finally, consistent with the data in LIRKO mice (Figure 1H), and human islet transplantation studies (Figure 4, B-D, and Supplemental Figure 8C), endogenous pancreatic islets from G15.5 NSG-LIRKO mice demonstrated a significant increase in $\beta$ cell proliferation (analyzed by BrdU, Ki67, or pHH3) compared with G15.5 NSG-Lox or G0 NSG-LIRKO mice (Supplemental Figure 9, A-C, and Supplemental Table 3). These results clearly point to stimulatory effects in response to increased insulin demand in both human and mouse islets.

Human ducts transplanted into NSG-LIRKO mice exhibit enhanced proliferation and presence of markers of $\beta$ cell differentiation. To further examine the translational relevance of our findings, we undertook an alternative transplantation approach and transplanted human pancreatic ductal "aggregates" together with human islets from the same donor (Figure 4A; for donor information, see Supplemental Table 2) in a ratio that mimics the pancreatic microenvironment. One striking observation in the duct cells was a significant increase in number of proliferating cells that was already evident in the nonpregnant (G0) NSG-LIRKO mice and increased even further at G15.5 (Figure 5, A and B). Further work is necessary to examine the consequences of this increased ductal proliferation. 


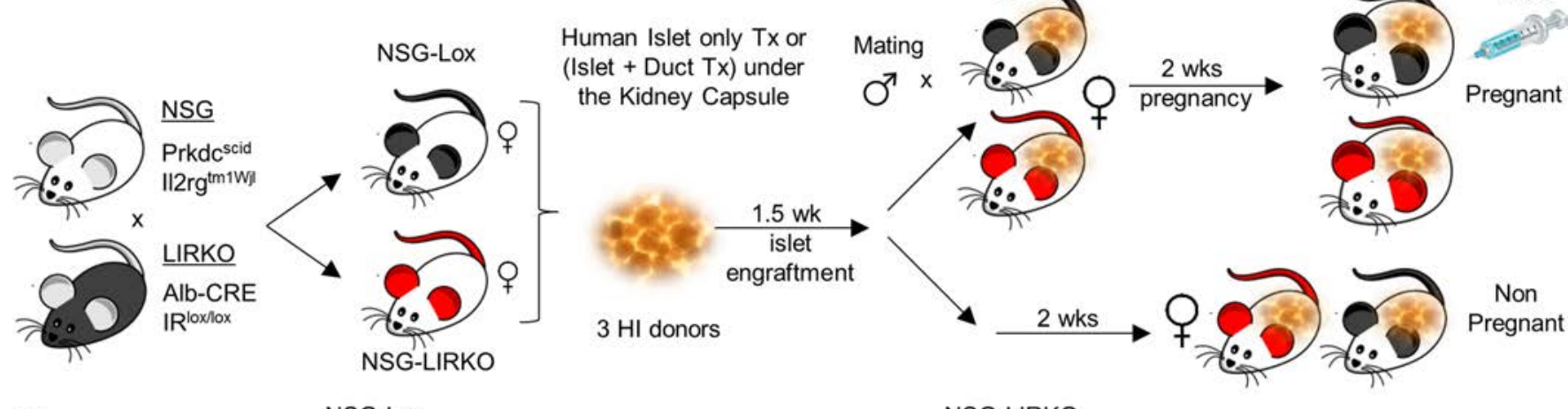

B
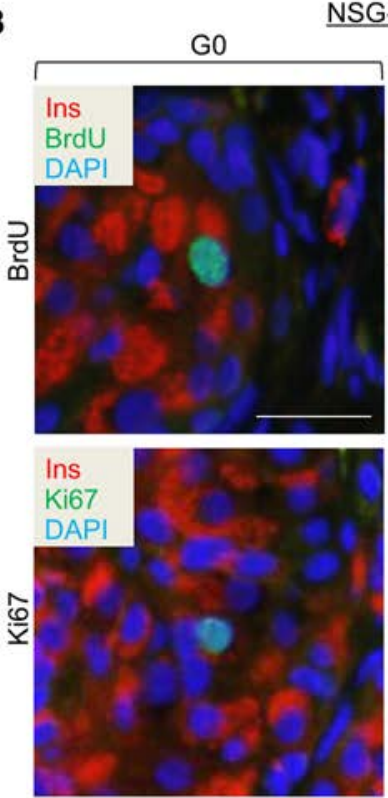

NSG-Lox
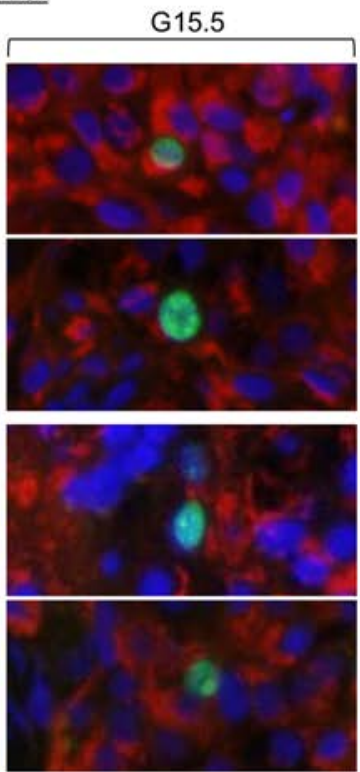

C

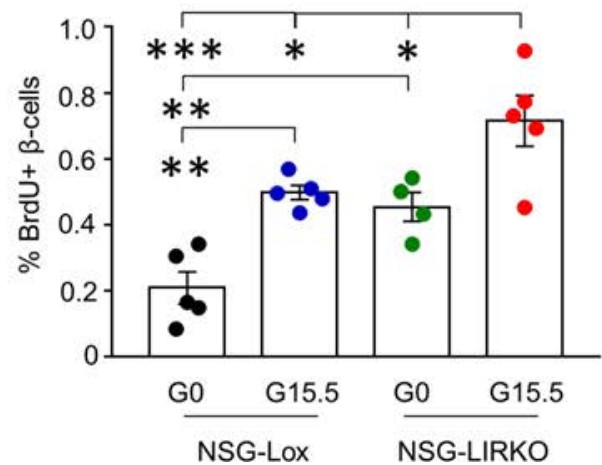

D

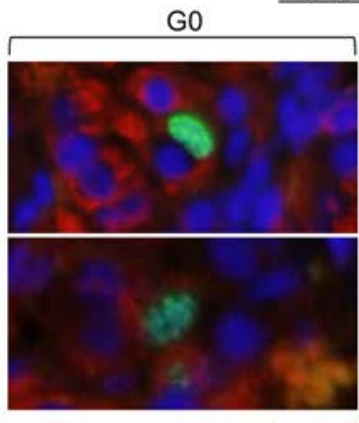

NSG-LIRKO

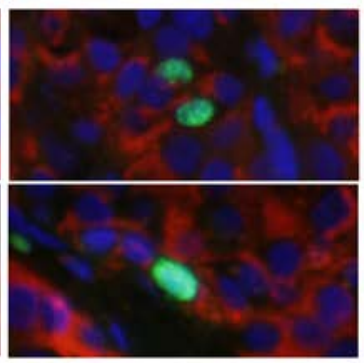

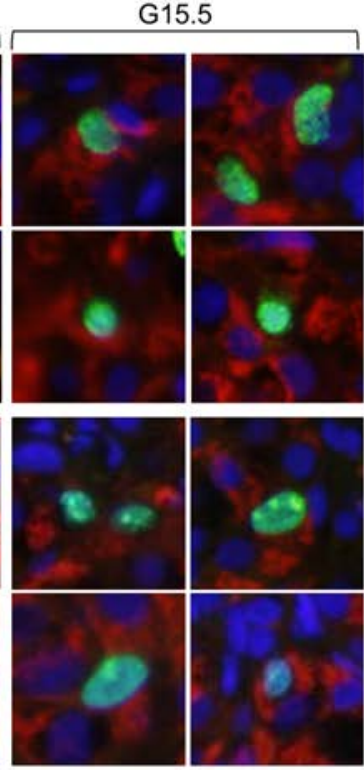

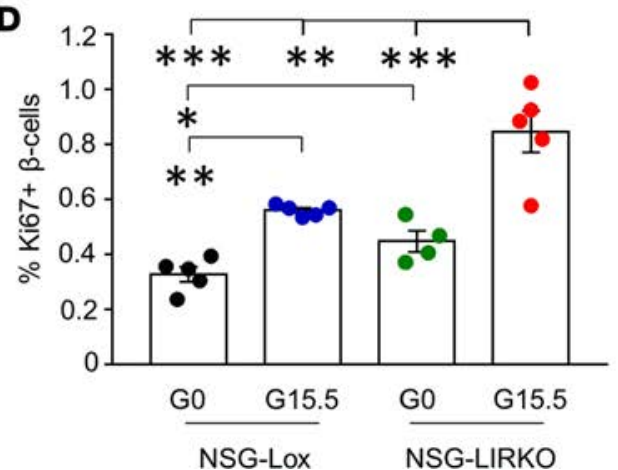

Figure 4. $\beta$ Cell proliferation is increased in response to pregnancy in human islets transplanted into NSG-LIRKO mice. (A) Experimental strategy showing NSC mice crossed with LIRKO mice to generate NSG-Lox and NSG-LIRKO female mice, followed by transplantation of human islets (1,000 islet equivalents [IEQ]) under the kidney capsule. Ten days after transplantation, mice were rendered pregnant and then sacrificed at G15.5 following BrdU injection. Nonpregnant mice transplanted with human islets were used as controls. Tx, transplant. (B) Representative immunofluorescence images of kidney sections obtained from pregnant and nonpregnant NSG-Lox and NSG-LIRKO mice with human islet transplants. Sections were immunostained for insulin (shown in red), BrdU (shown in green, upper), Ki67 (shown in green, lower), and nuclear marker DAPI (shown in blue). Scale bar: $25 \mu \mathrm{m}$. (C) Quantification of proliferating $\beta$ cells for BrdU and insulin ( $n=4-5$ mice per group, 1-way ANOVA) or (D) Ki67 and insulin ( $n=4-5$ mice per group, 1-way ANOVA) double-positive cells from human islet grafts from samples shown in B (for quantification, see Supplemental Table 3). Data are expressed as mean \pm SEM. ${ }^{*} P<0.05,{ }^{* *} P<0.01,{ }^{* * *} P<0.001$. 
To explore whether the compensatory increase in proliferating duct cells is a prelude to inducing differentiation toward the $\beta$ cell lineage, we immunostained the sections for key transcription factors known to be expressed during development of the endocrine pancreas (Figure 5C). Coimmunostaining for human duct-specific cytokeratin 19 (CK19) and transcription factor sex-determining region Y box 9 (SOX9) showed distinct nuclear staining for SOX9 in the NSG-Lox group while several duct cells were SOX9- the NSG-LIRKO group, implying some duct cells were differentiating toward the endocrine lineage (Figure 5D). This was supported by the presence of the endocrine progenitor marker neuronal differentiation (NeuroD1) in duct cells in pregnant NSG-LIRKO mice only (Figure 5E). Although human duct grafts in both NSG-Lox and NSG-LIRKO mice were virtually negative for paired box 6 (PAX6) staining, NSG-LIRKO (G0) mice demonstrated PAX6 ${ }^{+}$human duct cells that were more frequently detected in pregnant NSG-LIRKO mice (Figure 5F). The mature $\beta$ cell markers PDX1 and V-Maf Musculoaponeurotic Fibrosarcoma Oncogene Homolog A (MAFA) were observed more frequently in the pregnant NSG-LIRKO group (Figure 5, G and H). Finally, the percentage of insulin and CK19 double-positive cells was significantly higher in pregnant NSG-LIRKO mice compared with nonpregnant NSG-LIRKO mice and pregnant NSG-Lox animals (Figure 5, I and J). These results, from the first study to our knowledge where human ductal cells and islets were cotransplanted into mice and tracked for duct to endocrine cell change during pregnancy, indicate that increased insulin demand drives human ductal cells toward a $\beta$ or $\beta$-like cell identity.

Insulin ${ }^{+}$duct cells and small islet clusters are increased in human pregnancy and patients with T2D. To obtain further translational evidence for a ductal source of $\beta$ cells, we screened pancreas sections from human control and pregnant (physiological insulin resistance) samples as well as female patients with T2D (pathological insulin resistance) obtained from the Network for Pancreatic Organ Donors (nPOD) (for donor information, see Supplemental Table 2; because of the limited number of pregnant patient samples at nPOD, we decided to match the BMI of the patients with T2D cases with the pregnant cases because both are models of insulin resistance). Indeed, confocal microscopic analysis revealed a significant presence of insulin ${ }^{+}$ductal cells in both pregnant and T2D patients (Figure 6 , A and B). Quantification of islet clusters, as evidence of neogenesis (37), revealed an approximately 3 -fold increase in small clusters located close to ductal epithelium in both pregnant and T2D groups compared with controls (Figure 6, C and D). Moreover, while pancreatic islets adherent to CK19+ ductal cells tended to increase in pregnancy $(P=0.07)$, they reached statistical significance in $\mathrm{T} 2 \mathrm{D}$ samples (Figure 6, E and F). Interestingly, we observed insulin and glucagon double-positive clusters that appeared to be budding from ducts (Figure 6G), and CK19+ ducts were positive for multiple islet endocrine hormones (Figure $6 \mathrm{H}$ ) in both pregnant and female T2D cadaver samples, similar to a previous report on distal pancreatectomy in humans (41). Consistent with the mouse data (Figure 2D), analysis of pregnancy samples showed an increase in small islet clusters adjacent or closely localized to pancreatic ducts compared with nonpregnant controls (control $3.17 \%$ vs. pregnant $23.54 \%, P=$ 0.017) (Figure 6I). Finally, to provide additional evidence of $\beta$ cell proliferation in response to insulin resistance, we screened pancreas sections from human control, pregnant, and T2D patients obtained from nPOD (for donor information, see Supplemental Table 2). Although $\beta$ cell replication detected by Ki67 immunostaining was minimal in controls and virtually absent in T2D, the frequency was higher in pregnant patients during the last trimester. Although the total number of patients in the pregnancy group is limited and precludes statistical significance, it is notable that $\beta$ cell proliferation correlates with the duration of pregnancy (Supplemental Figure 10, A and B).

Presence of endocrine differentiation markers in ducts in pregnant and T2D patients. We next explored whether the ductal cells in sections from human cadaver pancreas showed presence of endocrine pancreatic differentiation markers similar to the experiment with the human islet and duct grafts (Figure 5, D-H). Coimmunostaining for human duct-specific CK19 and SOX9 showed nuclear staining for SOX9 in control cases while insulin-resistant samples showed ductal cells predominantly negative for SOX 9 (Figure 6J). Although we did not detect any $\mathrm{PDX}^{+}$or $\mathrm{PAX}^{+}$ductal cells in controls, they were clearly increased in insulin-resistant individuals (Figure 6, K and L). Finally, the mature $\beta$ cell marker, MAFA, was detected only in the ducts from the pregnant group (Figure 6M). These results suggest that, during increased insulin demand such as physiological or pathophysiological insulin resistance, ductal cells are mobilized to attain a $\beta$ cell identity. 
A

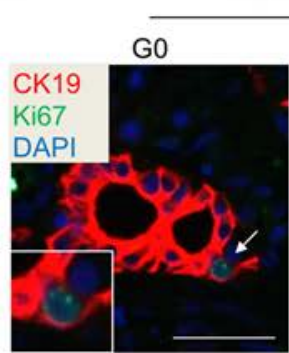

NSG-Lox

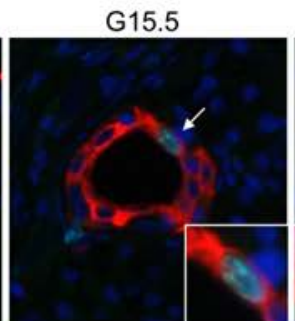

C

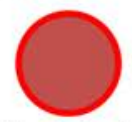

Pluripotent

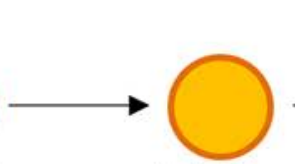

Endoderm

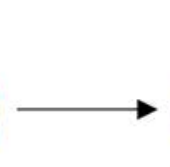

Pancreatic Endoderm

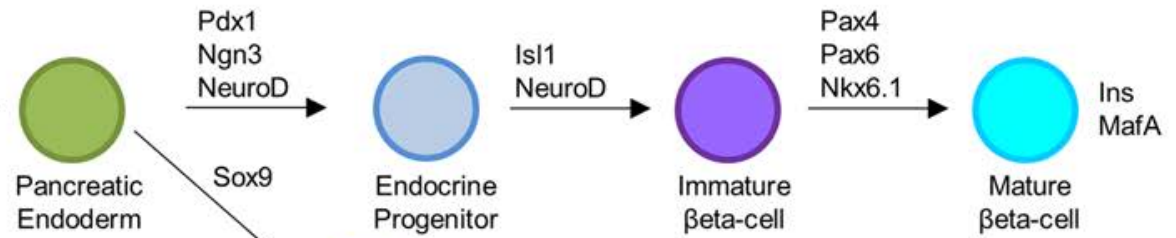

NSG-LIRKO
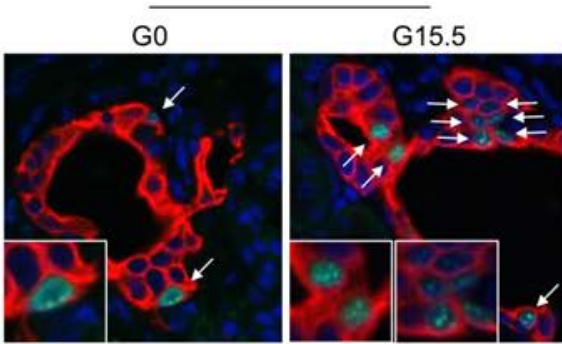
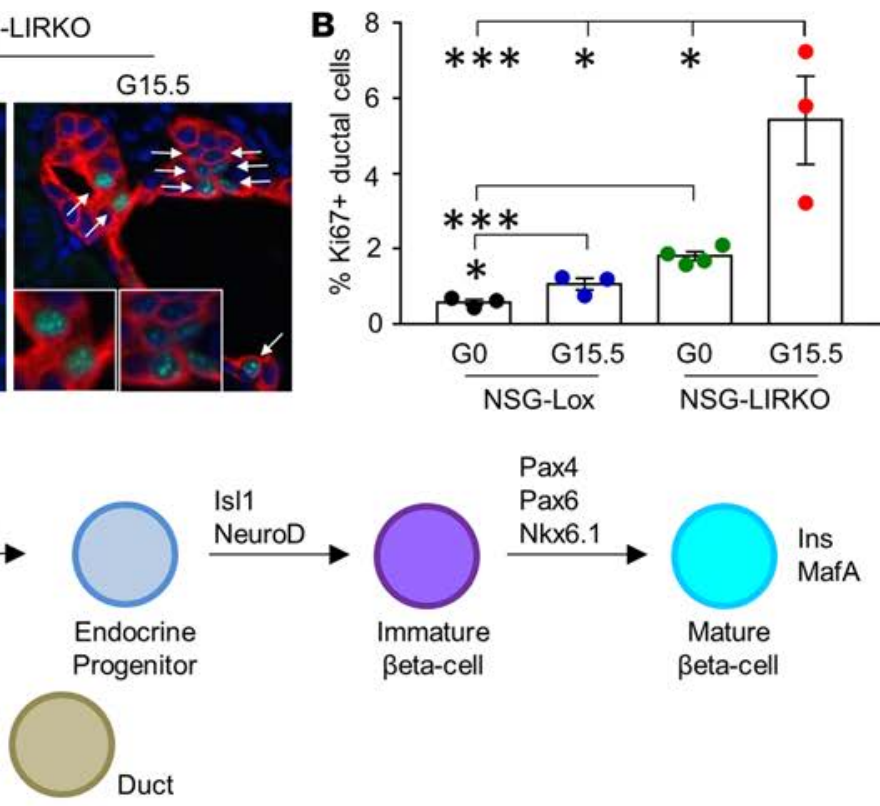

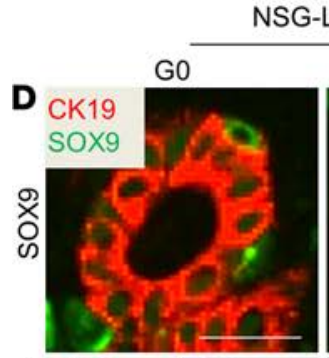

E CK19 NEUROD1 DAPI

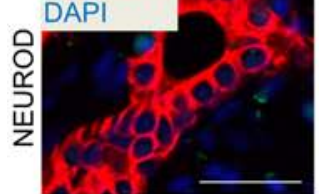

F CK19

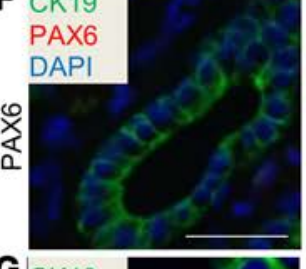

G $\overline{\text { CK19 }}$

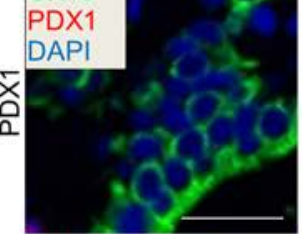

H $\overline{\text { CK19 }}$

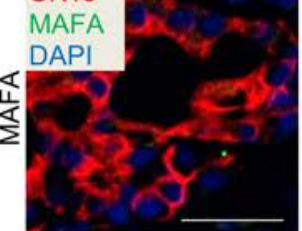

G15.5
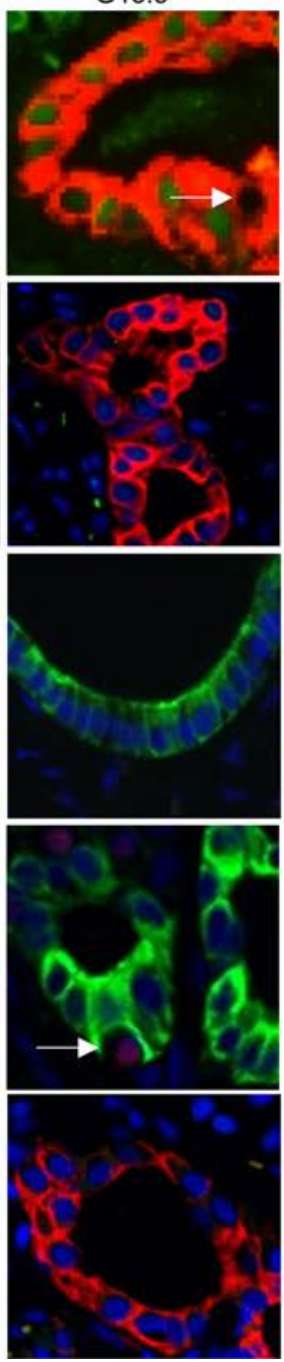
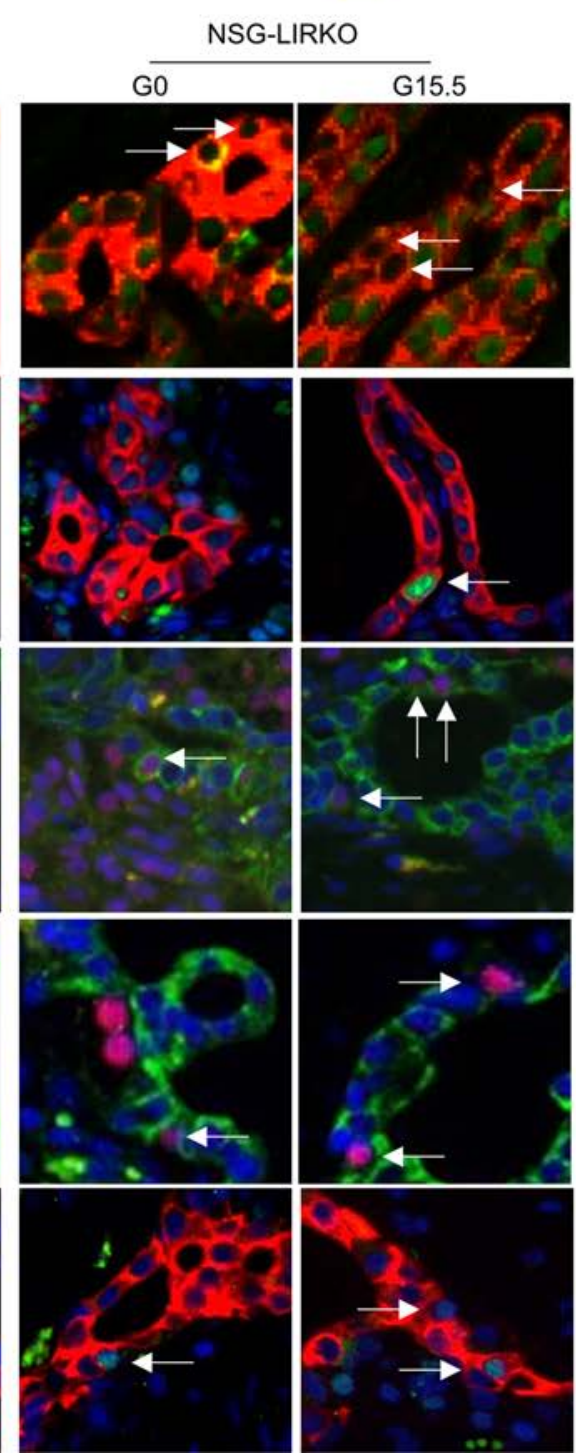
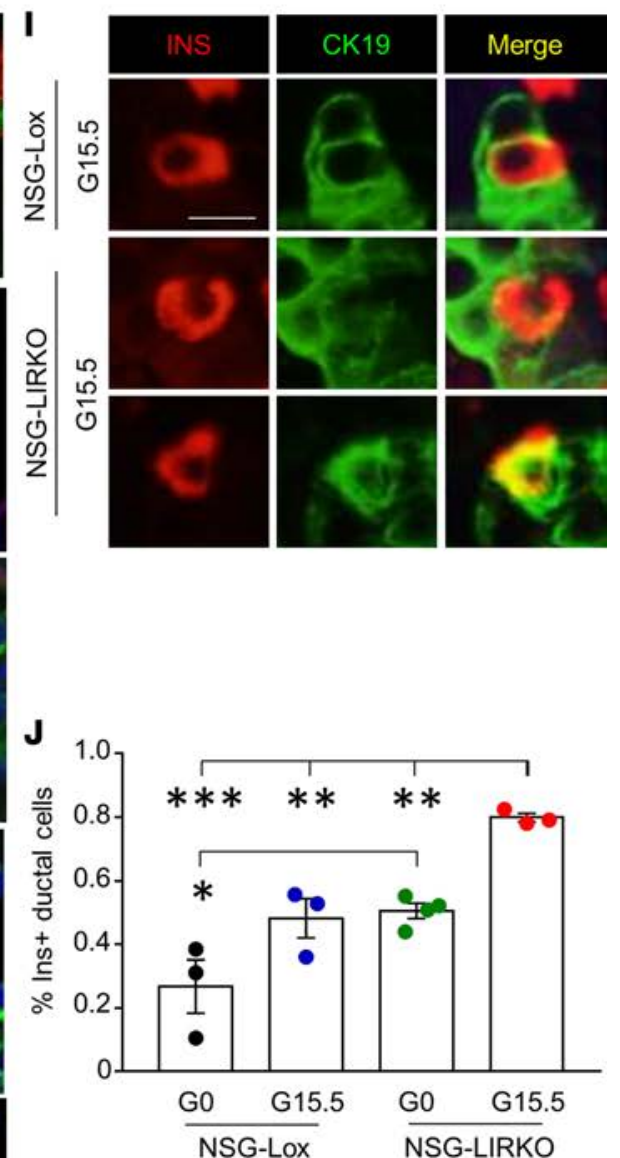
Figure 5. Enhanced proliferation and presence of markers of endocrine progenitors and mature $\beta$ cell markers in duct cells in response to pregnancy in NSG-LIRKO mice cotransplanted with human ducts and human islets. (A) Human islet and duct graft samples obtained from nonpregnant and pregnant NSG-Lox and NSG-LIRKO mice stained for CK19 and proliferation marker Ki67. White arrows show proliferating ductal cells. (B) Quantification of proliferating duct cells ( $n=3-4$ mice per group, 2-tailed Student's $t$ test). (C) An overview of development of $\beta$ cells showing key transcription factors. (D-H) Human islet and duct graft samples from A coimmunostained for CK19 and S0X9 (D), NeuroD1 (E), PAX6 (F), PDX1 (G), or MAFA (H). White arrows point to ductal cells positive for given markers. (I) Representative immunofluorescence staining of kidney graft sections obtained from pregnant and nonpregnant NSG-Lox and NSG-LIRKO mice transplanted with human islet and ducts showing insulin (colored red) and human duct marker CK19 (colored green) double-positive cells (merge, colored yellow). We transplanted 1,000 human IEQ with 100 ductal aggregates. (J) Insulin ${ }^{+}$ duct cells are expressed as percentage of total duct cells ( $n=3-4$ mice per group, 2-tailed Student's $t$ test). Scale bars: $25 \mu \mathrm{m}(\mathbf{A}-\mathbf{H}), 10 \mu \mathrm{m}$ (I). Data are expressed as mean \pm SEM. ${ }^{*} P<0.05,{ }^{* *} P<0.01$, and ${ }^{* *} P<0.001$.

\section{Discussion}

The key finding in this study is that neogenesis triggered by ductal epithelium contributes to the $\beta$ cell pool in response to a greater demand for insulin in physiological (pregnancy-related) or pathological (T2D-related) insulin-resistant states.

Young adult rodents undergo a rapid expansion of their $\beta$ cell mass via increased mitosis or hypertrophy during pregnancy. In addition to proliferation, some of the new $\beta$ cells arise from other pancreatic endocrine cells, such as glucagon-expressing $\alpha$ cells $(34,42)$ and somatostatin-expressing $\delta$ cells $(43)$; exocrine cells, such as acinar cells; or pancreatic ducts by transdifferentiation/neogenesis. The increased $\beta$ cell proliferation in nonpregnant LIRKO mice is, in part, because of the growth effect of the protease inhibitor, SerpinB1, which we reported recently (8). However, the further increase in $\beta$ cell mass/area at the end of pregnancy in LIRKO mice despite the relative reduction in proliferation from G.15.5 to P4 prompted us to investigate alternative sources and pathways contributing to the greater $\beta$ cell pool.

A striking observation was the increased number of insulin and glucagon double-positive duct cells along with the presence of small islet clusters, made up of insulin ${ }^{+}$or glucagon ${ }^{+}$cells positioned immediately adjacent to or very closely localized to ductal cells throughout the pancreas in pregnant LIRKO mice. An origin of endocrine cells from ductal epithelium was suggested several years ago (44) and reported in a case of partial pancreatectomy (45). Although the significantly reduced percentage of $\alpha$ and $\delta$ cells (Figure 1G) suggests that these islet cell types are a source of $\beta$ cell neogenesis as reported recently (43, 46), additional lineage-tracing studies are necessary to confirm this possibility. Our previous studies also suggest an association between insulin resistance and increased islet neogenesis in pancreas sections from insulin-resistant humans (47). Whether the decreased $\alpha$ cell proliferation observed in LIRKO mice also contributes to the $\beta$ cell pool by transdifferentiation requires further investigation.

Our understanding regarding the adaptive changes of $\beta$ cells during pregnancy is mostly based on rodent studies $(5,48)$, and the limited evidence supporting an increase in human $\beta$ cell area is derived from autopsy reports (3). Despite the low number of nPOD samples we examined, $\beta$ cell proliferation was relatively higher in human pregnant samples compared with controls. Our finding of enhanced $\beta$ cell proliferation in human islets transplanted into NSG-Lox mice in response to pregnancy, which is increased even further in NSG-LIRKO mice, indicates a dynamic reserve that is able to contribute to new $\beta$ cells. Indeed, the endogenous $\beta$ cell proliferation, assessed by 3 proliferation markers, clearly showed an increase in pregnant NSG-LIRKO mice, indicating that pregnancy and insulin resistance act synergistically in the $\beta$ cell compensation response. The absence of SOX9 expression in some ductal cells, especially in NSG-LIRKO mice transplanted with human duct cells, suggests an initial step in acquiring a $\beta$ cell identity. The transplantation studies show that duct cells expressing some of the endocrine markers were more distinct in pregnant NSG-LIRKO animals and support the observation that greater numbers of endocrine marker-positive duct cells emerge as insulin demand increases.

Although previous studies have reported alterations in replicating $\beta$ cell turnover and area in human pregnancy $(2,3)$, we report the presence of endocrine differentiation markers in pancreas sections from patients in different trimesters and provide translational relevance of the mouse studies. Furthermore, the significantly greater insulin ${ }^{+}$ductal cells, small islet clusters that are mainly localized close to ducts, and islets in close apposition to $\mathrm{CK} 19^{+}$duct cells indicate that neogenesis might also contribute to the $\beta$ cell compensation in physiological insulin-resistant states.

Examination of the pancreas sections from female T2D patients revealed a similar signature of endocrine progenitor markers. One intriguing finding was the absence of MAFA in the T2D pancreas sections. 

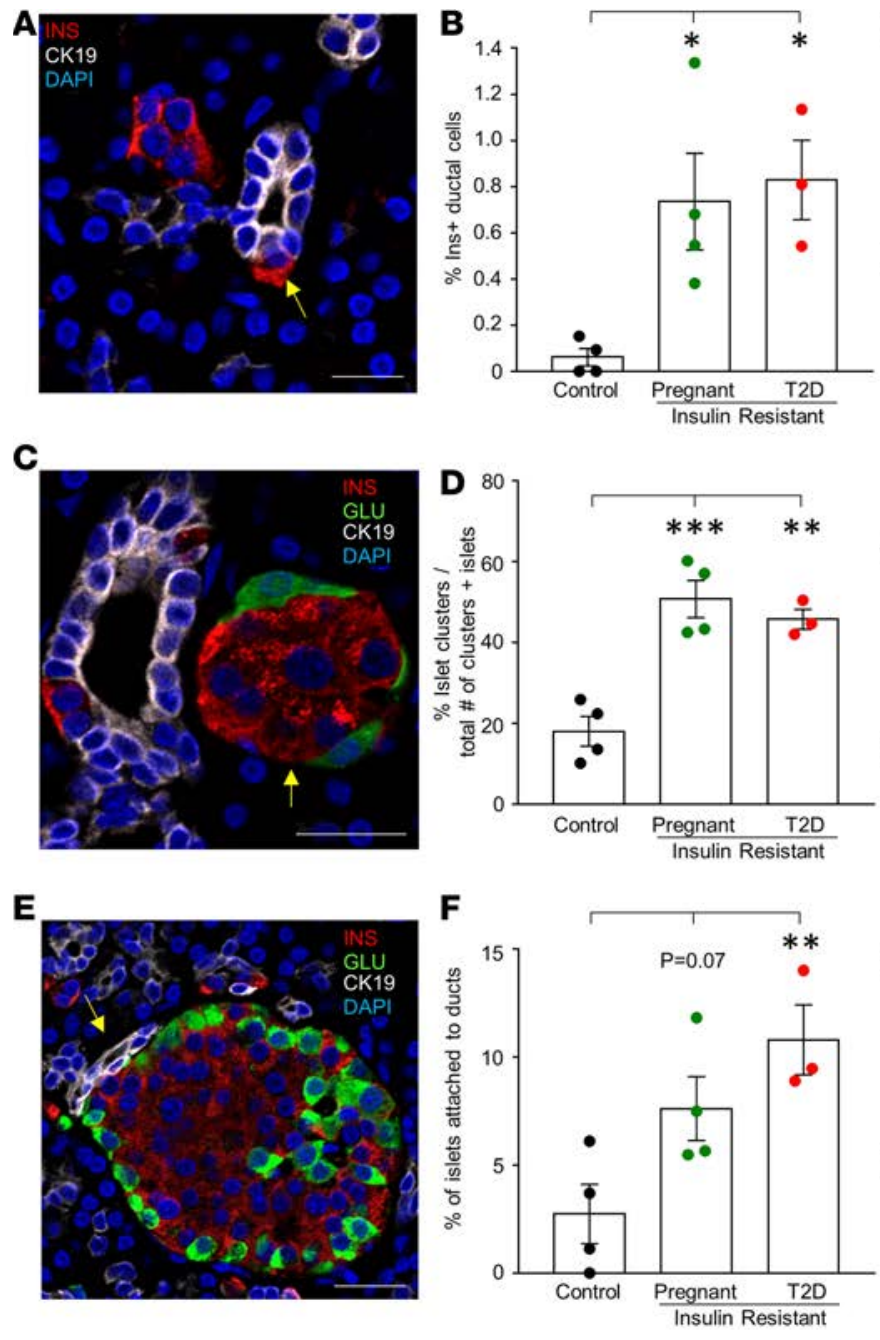
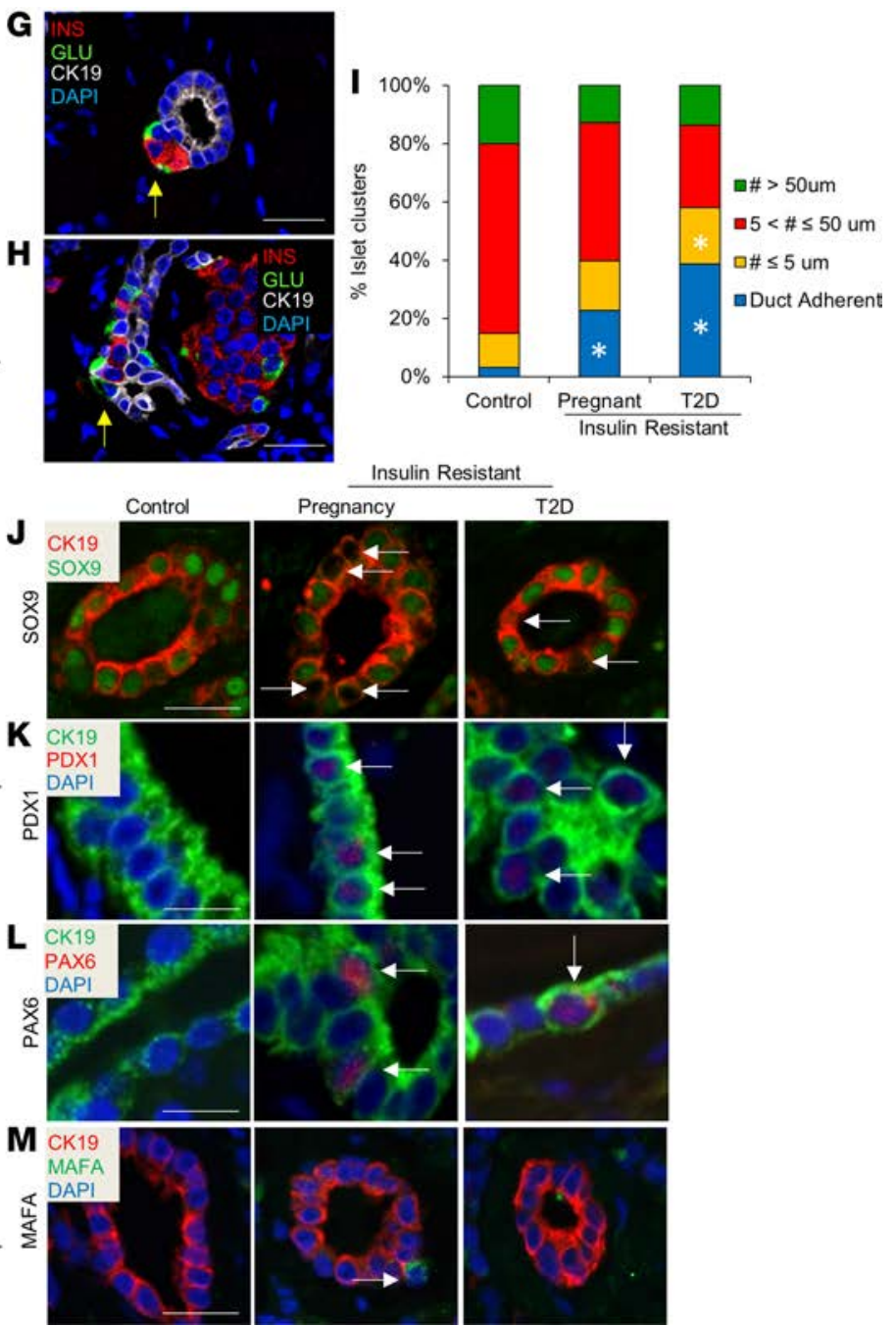

Figure 6. Presence of markers of endocrine progenitors and mature $\beta$ cells in duct cells and increased numbers of small islet clusters in human pregnant and T2D samples. (A and C) Confocal images of sections obtained from a 22-year-old female African American in the 40th week of pregnancy (nPOD 6383) immunostained for insulin (shown in red), glucagon (shown in green), CK19 (shown in white), and DAPI (shown in blue). (A) Insulin ${ }^{+}$ductal structures (marked by yellow arrow). (C) Small islet clusters (yellow arrow) expressing insulin and glucagon localized close to duct cells. (E) Confocal images of sections obtained from a 52-year-old female Hispanic/Latina with type 2 diabetes for 25 years (nPOD 6304) showing islets attached to CK19+ ductal cells (yellow arrow). (B, D, and F) Quantification of the percentage of (B) insulin ${ }^{+}$duct cells ( $n=3-4$ patients per group, 1-way ANOVA); (D) islet clusters expressed as percentage of islets plus small islet clusters ( $n=3-4$ patients per group, 1-way ANOVA); and (F) total islets attached to duct cells ( $n=3-4$ patients per group, 1-way ANOVA). (C) A small islet cluster (yellow arrow) that appears to be budding from the ductal structures (nPOD 6383). (H) Multiple endocrine hormone-positive ductal structures (yellow arrow) (nPOD 6304). (I) Quantification of the distance of islet clusters (<10 islet cells) from the ductal structures. Between 13 and 93 islet clusters were analyzed for each pancreatic section per sample (2-tailed Student's $t$ test). (J-M) Human pancreas sections coimmunostained for CK19 and SOX9 (J), PDX1 (K), PAX6 (L), or MAFA (M). White arrows point to ductal cells positive for given markers. Scale bars: $25 \mu \mathrm{m}$. Data are expressed as mean \pm SEM. ${ }^{*} P<0.05,{ }^{* *} P<0.01$, and ${ }^{* * *} P<0.001$.

Previous studies have reported that stress conditions have the potential to alter $\beta$ cell-enriched transcription factors, including $M A F A$, as well as alterations in their nuclear versus cytosolic localization (49-52). This may explain, in part, the absence of nuclear MAFA expression in pancreas sections from T2D patients while expression remained in human ducts that were obtained from controls without diabetes and transplanted into mice. Alternatively, this may be an example of heterogeneity that is observed in pathological conditions affecting the pancreas (53).

Taken together, we report the use of lineage tracing, human duct/islet engraftment in mouse models, and immunohistochemical analyses of cadaveric pancreata to demonstrate the existence of a dynamic source of $\beta$ cell mass derived from human pancreatic ducts in the adaptive compensatory response to insulin-resistant states. These findings highlight the duct as a potential target to mobilize conversion of non- $\beta$ cells to functional $\beta$ cells for treating patients with diabetes. 


\section{Methods}

Mice. Mice were housed on a 12-hour light/12-hour dark cycle with water and food ad libitum. Only female mice were used and the age of the mice is specified for each experiment. Ins $R^{\mathrm{f} / \mathrm{fl}}$ mice were used as control animals (C57BL/6J background, a gift from C.R. Kahn of Joslin Diabetes Center). Alb-Cre InsR ${ }^{\mathrm{f} / / \mathrm{fl}}$ (LIRKO, a gift from C.R. Kahn of Joslin Diabetes Center) mice (40) and CAII-CreER ${ }^{\mathrm{TM}} R 26 R$-eYFP mice (a gift from S. Bonner-Weir of Joslin Diabetes Center) (38) were bred to generate Lox-YFP or LIRKO-YFP animals for lineage-tracing experiments. NSG mice were purchased from The Jackson Laboratory and were bred with LIRKO mice to generate NSG-Lox or NSG-LIRKO mice. Pregnancy was confirmed by the presence of a vaginal plug and designated day 0.5 of gestation (G0.5). For TM-inducible eYFP expression, mice were treated with $100 \mathrm{mg} / \mathrm{kg}$ BW of TM (MilliporeSigma) dissolved in corn oil (MilliporeSigma) and injected i.p. for 3 consecutive days starting at pregnancy day 10.5. Body weights and nonfasted blood glucose were measured weekly. Blood was collected before (G0), during (G15.5 and G17.5), and after (P0 and P4) pregnancy for measurements of serum insulin, C-peptide, and pregnancy hormones (by ELISA).

Islet morphology, immunohistochemistry, and electron microscopy. Mouse pancreata and islet-bearing kidney sections were harvested, fixed, and embedded in paraffin. Sections were stained using antibodies against Ki67 (Dako, M7240, 1:100), BrdU (Dako, M0744, 1:100), pHH3 (MilliporeSigma, 06-570, 1:250), insulin (Abcam, ab7842, 1:500), non- $\beta$ cell hormones glucagon (MilliporeSigma, G2654, 1:10,000) and somatostatin (Abcam, ab103709, 1:500), serotonin (Immunostar, 20080, 1:200), $\beta$-catenin (BD Biosciences, 610154, 1:200) or pancreatic duct marker DBA (Vevtor, FL-1031, 1:100), YFP (Abcam, ab38689, 1:200), CK19 (Abcam, ab7754, 1:200), SOX9 (MilliporeSigma, ab5535, 1:100), PDX1 (Abcam, ab134150, 1:1,000) NeuroD1 (Abcam, ab60704, 1:100), PAX6 (Santa Cruz, sc81649, 1:50), MAFA (Abcam, ab26405, 1:200), and TUNEL (ApopTag, Chemicon, S7100) and counterstained with DAPI (MilliporeSigma, D9564, 1:6,600). For estimation of $\beta$ cell proliferation, 1,000-7,000 $\beta$ cell nuclei were counted per animal, and data were expressed as percentage of $\mathrm{Ki}^{+} 7^{+}, \mathrm{BrdU}^{+}$or $\mathrm{pHH} 3^{+} \beta$ cells. $\beta$ Cell mass was evaluated by point-counting morphometry on immunofluorescence-stained sections of pancreas as described previously (40). For transmission electron microscopy, islets were processed by the Joslin Advanced Microscopy Core (Joslin Diabetes Research Center) facility as reported previously (54).

Human islet and duct transplantation studies. Upon receipt, human islets were cultured overnight in Miami Media 1A (Cellgro). Hand-picked islets (1,000 islet equivalents [IEQ]) were transplanted either alone or with 100 human ductal aggregates (cluster of ductal cells) under the kidney capsule of both NSG-Lox and NSG-LIRKO mice as described previously $(55,56)$. After allowing 10 days after transplantation for islet engraftment, mice were either maintained in a nonpregnant state or allowed to breed to generate pregnant mice, which were injected with BrdU (100 mg/kg BW) for 3 days and sacrificed 6 hours after receiving the final BrdU injection at pregnancy day 15.5. Blood was collected on the day of tissue harvesting for the measurement of human insulin and C-peptide.

Human pancreas sections. Human pancreas sections (for human donor information, see Supplemental Table 2) were immunostained using antibodies against insulin (Abcam, ab7842, 1:500), glucagon (MilliporeSigma, G2654, 1:10,000), CK19 (Abcam, ab15463, 1:200), and DAPI (MilliporeSigma, D9564, 1:6,600).

Glucose and insulin tolerance tests, in vitro and in vivo insulin and serotonin secretion, and insulin and serotonin content. Glucose and insulin tolerance tests and acute insulin secretion tests and insulin secretion during GTT were performed as described previously (57). To avoid injury to embryos during i.p. injections, we used oral gavage for glucose tolerance tests. For in vitro insulin and serotonin secretion tests, following overnight culture and 2-hour starvation, 20 size-matched islets were preincubated in Krebs-Ringer bicarbonate buffer for 1 hour at low glucose $(3.3 \mathrm{mM})$, and the medium was changed to high glucose $(16.7 \mathrm{mM})$ to stimulate islets for 1 additional hour. Medium was collected for insulin and serotonin ELISA, and the content of insulin and serotonin was measured by acid ethanol extraction followed by ELISA (57).

Quantification of islet clusters. As previously described (37), pancreatic sections from mouse or human cadaver samples were immunostained for islet endocrine cell hormones and were manually counted by a single observer, and islets having fewer than 8 to 10 endocrine cells were considered scattered islets. The clusters were further analyzed according to the distance from the pancreatic ducts by using ImageJ software (NIH).

Statistics. All data are expressed as mean \pm SEM. Statistical significance was determined by 2-tailed Student's $t$ test and 1-way ANOVA (followed by the Tukey's and Dunnett's multiple comparisons tests; GraphPad Prism 7) test among groups. $P$ value of less than 0.05 was considered to represent a significant difference. 
Study approval. All mice experiments were conducted at Joslin Diabetes Center with approval of its Institutional Animal Care and Use Committee and were in accordance with NIH guidelines. Human islets were obtained from the Integrated Islet Distribution Program or Prodo Laboratories (Supplemental Table 2). Cadaveric pancreas sections were obtained from the nPOD (Supplemental Table 2). All studies and protocols used were approved by the Joslin Diabetes Center's Committee on Human Studies (CHS\#5-05).

\section{Author contributions}

ED conceived the idea, designed and performed the experiments, analyzed the data, and wrote the manuscript. DFDJ, SK, GB, RWSN, AEO, and AKKT researched data and provided technical support or critical discussions of the manuscript. SB and JH performed immunohistochemistry. RNK conceived the idea, designed the experiments, supervised the project, and wrote the manuscript. All the authors have reviewed the manuscript.

\section{Acknowledgments}

LIRKO mice were provided by C.R. Kahn (Joslin Diabetes Center). We thank S. Bonner-Weir (Joslin Diabetes Center) for discussions regarding duct cell transplantations and providing the CAII-CreER R26R-eYFP mice. We are grateful to D. Greiner (University of Massachusetts) for advice on generating NSG-LIRKO mice. We thank C. Cahill (Joslin Advanced Microscopy Core) for assistance with confocal and electron microscopy; L. Kannan, Z. Fu, and G. Sankaranarayanan (Joslin Diabetes Center) for ELISAs; and J. Hollister-Lock (Joslin Diabetes Center) for assistance with mouse islet isolations. This research was performed with the support of the nPOD, a collaborative type 1 diabetes research project sponsored by the Juvenile Diabetes Research Foundation. Organ procurement organizations partnering with nPOD to provide research resources are listed at http://www.jdrfnpod.org/for-partners/ npod-partners/. ED was supported by a Senior Juvenile Diabetes Research Foundation Fellowship (JDRF-3-APF-2014-220-A-N). This project was funded by NIH R01 DK67536 (to RNK) and in part by NIH R01 DK103215 (to RNK).

Address correspondence to: Rohit N. Kulkarni, Section of Islet Cell and Regenerative Biology, Joslin Diabetes Center, One Joslin Place, Boston, 02215 Massachusetts, USA. Phone: 617.309.3460; Email: Rohit. Kulkarni@joslin.harvard.edu.

1. Parsons JA, Brelje TC, Sorenson RL. Adaptation of islets of Langerhans to pregnancy: increased islet cell proliferation and insulin secretion correlates with the onset of placental lactogen secretion. Endocrinology. 1992;130(3):1459-1466.

2. Van Assche FA, Aerts L, De Prins F. A morphological study of the endocrine pancreas in human pregnancy. Br J Obstet Gynae col. 1978;85(11):818-820.

3. Butler AE, et al. Adaptive changes in pancreatic beta cell fractional area and beta cell turnover in human pregnancy. Diabetologia. 2010;53(10):2167-2176.

4. Scaglia L, Smith FE, Bonner-Weir S. Apoptosis contributes to the involution of beta cell mass in the post partum rat pancreas. Endocrinology. 1995;136(12):5461-5468.

5. Rieck S, et al. The transcriptional response of the islet to pregnancy in mice. Mol Endocrinol. 2009;23(10):1702-1712

6. De Jesus DF, Kulkarni RN. Epigenetic modifiers of islet function and mass. Trends Endocrinol Metab. 2014;25(12):628-636.

7. El Ouaamari A, et al. SerpinB1 promotes pancreatic $\beta$ cell proliferation. Cell Metab. 2016;23(1):194-205.

8. El Ouaamari A, et al. Liver-derived systemic factors drive $\beta$ cell hyperplasia in insulin-resistant states. Cell Rep. 2013;3(2):401-410.

9. Parsons JA, Bartke A, Sorenson RL. Number and size of islets of Langerhans in pregnant, human growth hormone-expressing transgenic, and pituitary dwarf mice: effect of lactogenic hormones. Endocrinology. 1995;136(5):2013-2021.

10. Rieck S, Kaestner KH. Expansion of $\beta$-cell mass in response to pregnancy. Trends Endocrinol Metab. 2010;21(3):151-158.

11. Vasavada RC, Gonzalez-Pertusa JA, Fujinaka Y, Fiaschi-Taesch N, Cozar-Castellano I, Garcia-Ocaña A. Growth factors and beta cell replication. Int J Biochem Cell Biol. 2006;38(5-6):931-950.

12. Freemark M. Regulation of maternal metabolism by pituitary and placental hormones: roles in fetal development and metabolic programming. Horm Res. 2006;65 Suppl 3:41-49.

13. Gupta RK, et al. Expansion of adult $\beta$-cell mass in response to increased metabolic demand is dependent on HNF-4 $\alpha$. Genes Dev. 2007;21(7):756-769.

14. Johansson M, Mattsson G, Andersson A, Jansson L, Carlsson PO. Islet endothelial cells and pancreatic $\beta$-cell proliferation: studies in vitro and during pregnancy in adult rats. Endocrinology. 2006;147(5):2315-2324.

15. Brelje TC, Stout LE, Bhagroo NV, Sorenson RL. Distinctive roles for prolactin and growth hormone in the activation of signal transducer and activator of transcription 5 in pancreatic islets of langerhans. Endocrinology. 2004;145(9):4162-4175.

16. Karnik SK, et al. Menin controls growth of pancreatic $\beta$-cells in pregnant mice and promotes gestational diabetes mellitus. Science. 2007;318(5851):806-809.

17. Kim H, et al. Serotonin regulates pancreatic $\beta$ cell mass during pregnancy. Nat Med. 2010;16(7):804-808. 
18. Stewart AF, et al. Human $\beta$-cell proliferation and intracellular signaling: part 3. Diabetes. 2015;64(6):1872-1885.

19. Mashima H, et al. Betacellulin and activin A coordinately convert amylase-secreting pancreatic AR42J cells into insulin-secreting cells. J Clin Invest. 1996;97(7):1647-1654.

20. Zhou Q, Brown J, Kanarek A, Rajagopal J, Melton DA. In vivo reprogramming of adult pancreatic exocrine cells to $\beta$-cells. Nature. 2008;455(7213):627-632.

21. Clayton HW, et al. Pancreatic inflammation redirects acinar to $\beta$ cell reprogramming. Cell Rep. 2016;17(8):2028-2041.

22. Desai BM, et al. Preexisting pancreatic acinar cells contribute to acinar cell, but not islet $\beta$ cell, regeneration. J Clin Invest. 2007;117(4):971-977.

23. Bonner-Weir S, et al. The pancreatic ductal epithelium serves as a potential pool of progenitor cells. Pediatr Diabetes. 2004;5(suppl 2):16-22.

24. Dor Y, Brown J, Martinez OI, Melton DA. Adult pancreatic $\beta$-cells are formed by self-duplication rather than stem-cell differentiation. Nature. 2004;429(6987):41-46.

25. Teta M, Rankin MM, Long SY, Stein GM, Kushner JA. Growth and regeneration of adult $\beta$ cells does not involve specialized progenitors. Dev Cell. 2007;12(5):817-826.

26. Xiao X, et al. No evidence for $\beta$ cell neogenesis in murine adult pancreas. J Clin Invest. 2013;123(5):2207-2217.

27. Blaine SA, Ray KC, Anunobi R, Gannon MA, Washington MK, Means AL. Adult pancreatic acinar cells give rise to ducts but not endocrine cells in response to growth factor signaling. Development. 2010;137(14):2289-2296.

28. Inada A, et al. Carbonic anhydrase II-positive pancreatic cells are progenitors for both endocrine and exocrine pancreas after birth. Proc Natl Acad Sci U S A. 2008;105(50):19915-19919.

29. Solar M, et al. Pancreatic exocrine duct cells give rise to insulin-producing $\beta$ cells during embryogenesis but not after birth. Dev Cell. 2009;17(6):849-860.

30. Cano DA, et al. Regulated $\beta$-cell regeneration in the adult mouse pancreas. Diabetes. 2008;57(4):958-966.

31. Jones LC, Clark A. $\beta$-Cell neogenesis in type 2 diabetes. Diabetes. 2001;50(suppl 1):S186-S187.

32. Kopp JL, et al. Sox $9^{+}$ductal cells are multipotent progenitors throughout development but do not produce new endocrine cells in the normal or injured adult pancreas. Development. 2011;138(4):653-665.

33. Pan FC, et al. Spatiotemporal patterns of multipotentiality in Ptfla-expressing cells during pancreas organogenesis and injury-induced facultative restoration. Development. 2013;140(4):751-764.

34. Thorel F, et al. Conversion of adult pancreatic $\alpha$-cells to $\beta$-cells after extreme $\beta$-cell loss. Nature. 2010;464(7292):1149-1154.

35. Okada T, et al. Insulin receptors in $\beta$-cells are critical for islet compensatory growth response to insulin resistance. Proc Natl Acad Sci U S A. 2007;104(21):8977-8982.

36. Kahraman S, Dirice E, De Jesus DF, Hu J, Kulkarni RN. Maternal insulin resistance and transient hyperglycemia impact the metabolic and endocrine phenotypes of offspring. Am J Physiol Endocrinol Metab. 2014;307(10):E906-E918.

37. Xu G, Stoffers DA, Habener JF, Bonner-Weir S. Exendin-4 stimulates both $\beta$-cell replication and neogenesis, resulting in increased $\beta$-cell mass and improved glucose tolerance in diabetic rats. Diabetes. 1999;48(12):2270-2276.

38. Wu F, et al. TNF-like weak inducer of apoptosis (TWEAK) promotes beta cell neogenesis from pancreatic ductal epithelium in adult mice. PLoS One. 2013;8(8):e72132.

39. Shultz LD, et al. Humanized NOD/LtSz-scid IL2 receptor common $\gamma$ chain knockout mice in diabetes research. Ann $N Y$ Acad Sci. 2007;1103:77-89.

40. Michael MD, et al. Loss of insulin signaling in hepatocytes leads to severe insulin resistance and progressive hepatic dysfunction. Mol Cell. 2000;6(1):87-97.

41. Patti ME, et al. Severe hypoglycaemia post-gastric bypass requiring partial pancreatectomy: evidence for inappropriate insulin secretion and pancreatic islet hyperplasia. Diabetologia. 2005;48(11):2236-2240.

42. Collombat $P$, et al. The ectopic expression of Pax4 in the mouse pancreas converts progenitor cells into $\alpha$ and subsequently $\beta$ cells. Cell. 2009;138(3):449-462.

43. Druelle N, et al. Ectopic expression of Pax4 in pancreatic $\delta$ cells results in $\beta$-like cell neogenesis. J Cell Biol. 2017;216(12):4299-4311.

44. Bensley RR. Studies on the pancreas of the Guinea Pig. Am J Anat. 1911;12(3):297-388.

45. Bonner-Weir S, Li WC, Ouziel-Yahalom L, Guo L, Weir GC, Sharma A. $\beta$-Cell growth and regeneration: replication is only part of the story. Diabetes. 2010;59(10):2340-2348.

46. Ben-Othman N, et al. Long-term GABA administration induces $\alpha$ cell-mediated $\beta$-like cell neogenesis. Cell. 2017;168(1-2):73-85.e11.

47. Mezza T, et al. Insulin resistance alters islet morphology in nondiabetic humans. Diabetes. 2014;63(3):994-1007.

48. Ernst S, Demirci C, Valle S, Velazquez-Garcia S, Garcia-Ocaña A. Mechanisms in the adaptation of maternal $\beta$-cells during pregnancy. Diabetes Manag (Lond). 2011;1(2):239-248.

49. Bonnavion R, et al. Both PAX4 and MAFA are expressed in a substantial proportion of normal human pancreatic $\alpha$ cells and deregulated in patients with type 2 diabetes. PLoS One. 2013;8(8):e72194.

50. Matsuoka TA, et al. Preserving Mafa expression in diabetic islet $\beta$-cells improves glycemic control in vivo. J Biol Chem. 2015;290(12):7647-7657.

51. Guo S, et al. Inactivation of specific $\beta$ cell transcription factors in type 2 diabetes. J Clin Invest. 2013;123(8):3305-3316.

52. Cinti F, et al. Evidence of $\beta$-cell dedifferentiation in human type 2 diabetes. J Clin Endocrinol Metab. 2016;101(3):1044-1054.

53. Patti ME, et al. Heterogeneity of proliferative markers in pancreatic $\beta$-cells of patients with severe hypoglycemia following Roux-en-Y gastric bypass. Acta Diabetol. 2017;54(8):737-747.

54. Liew CW, et al. The pseudokinase tribbles homolog 3 interacts with ATF4 to negatively regulate insulin exocytosis in human and mouse beta cells. J Clin Invest. 2010;120(8):2876-2888.

55. Dirice E, et al. Inhibition of DYRK1A stimulates human $\beta$-cell proliferation. Diabetes. 2016;65(6):1660-1671.

56. Dhawan S, Dirice E, Kulkarni RN, Bhushan A. Inhibition of TGF- $\beta$ signaling promotes human pancreatic $\beta$-cell replication. Diabetes. 2016;65(5):1208-1218.

57. Kulkarni RN, et al. Altered function of insulin receptor substrate-1-deficient mouse islets and cultured $\beta$-cell lines. J Clin Invest. 1999;104(12):R69-R75 